\title{
On the Codes over a Semilocal Finite Ring
}

\author{
Abdullah Dertli \\ Department of Mathematics \\ Ondokuz Mayıs University \\ Samsun, Turkey
}

\author{
Yasemin Cengellenmis \\ Department of Mathematics \\ Trakya University \\ Edirne, Turkey
}

\author{
Senol Eren \\ Department of Mathematics \\ Ondokuz Mayıs University \\ Samsun, Turkey
}

\begin{abstract}
In this paper, we study the structure of cyclic, quasi cyclic, constacyclic codes and their skew codes over the finite ring R. The Gray images of cyclic, quasi cyclic, skew cyclic, skew quasi cyclic and skew constacyclic codes over $\mathbf{R}$ are obtained. A necessary and sufficient condition for cyclic (negacyclic) codes over $\mathbf{R}$ that contains its dual has been given. The parameters of quantum error correcting codes are obtained from both cyclic and negacyclic codes over R. Some examples are given. Firstly, quasi constacyclic and skew quasi constacyclic codes are introduced. By giving two inner product, it is investigated their duality. A sufficient condition for 1 generator skew quasi constacyclic codes to be free is determined.
\end{abstract}

Keywords-Cyclic codes; Skew cyclic codes; Quantum codes

\section{INTRODUCTION}

In the beginning, a lot of research on error-correcting codes are concentrated on codes over finite fields. Since the revelation in 1994 [17], there has been a lot of interest in codes over finite rings. The structure of a certain type of codes over many rings are determined such as negacyclic, cyclic, quasi-cyclic, consta cyclic codes in $[6,11,20,21,22,23,26,32]$. Many methods and many approaches are applied to produce certain types of codes with good parameters and properties.

Some authors generalized the notion of cyclic, quasi-cyclic and constacyclic codes by using generator polynomials in skew polynomial rings $[1,2,5,7,8,9,14,15,18,27,30]$.

Moreover, in [10] Calderbank et al. gave a way to construct quantum error correcting codes from the classical error-correcting codes, although the theory of quantum errorcorrecting codes has striking differences from the theory of classical error correcting codes. Many good quantum codes have been constructed by using classical cyclic codes over finite fields or finite rings with self orthogonal (or dual containing) properties in $[3,12,13,16,19,24,25,28,29,31]$.

In [4] they introduced the finite ring $R=Z_{3}[v] /\left\langle v^{3}-v\right\rangle$. They studied the structure of this ring. The algebraic structure of cyclic and dual codes was also studied. A MacWilliams type identity was established.

In this paper, first of all we gave some definitions. By giving the duality of codes via inner product, it is shown that $C$ is self orthogonal code over $R$, so is $\phi(C)$, where $\phi$ is a Gray map.

The Gray images of cyclic and quasi-cyclic codes over $\mathrm{R}$ are obtained. A linear code over $\mathrm{R}$ is represented using three ternary codes and the generator matrix is given.
After a cyclic (negacyclic) code over $\mathrm{R}$ is represented via cyclic (negacyclic) codes over $Z_{3}$, it is determined the dual of cyclic (negacyclic) code. A necessary and sufficient condition for cyclic (negacyclic) code over $\mathrm{R}$ that contains its dual is given. The parameters of quantum error-correcting codes are obtained from both cyclic and negacyclic codes over R. As a last, some examples are given about quantum error-correcting codes.

When $\mathrm{n}$ is odd, it is defined the $\lambda$-constacyclic codes over $\mathrm{R}$ where $\lambda$ is unit. A constacyclic code is represented using either cyclic codes or negacyclic codes of length $n$.

It is found the nontrivial automorphism $\theta$ on the ring $\mathrm{R}$. By using this automorphism, the skew cyclic, skew quasicyclic and skew constacyclic codes over $\mathrm{R}$ are introduced. The number of distinct skew cyclic codes over $\mathrm{R}$ is given. The Gray images of skew codes are obtained.

Firstly, quasi-constacyclic and skew quasi-constacyclic codes over $\mathrm{R}$ are introduced. By using two inner product, it is investigated the duality about quasi-constacyclic and skew quasi-constacyclic codes over R. The Gray image of skew quasi-constacyclic codes over $\mathrm{R}$ is determined. A sufficient condition for 1-generator skew quasi-constacyclic code to be free is determined.

\section{PRELIMINARIES}

Suppose $R=Z_{3}+v Z_{3}+v^{2} Z_{3}$ where $v^{3}=v$ and $Z_{3}=$ $\{0,1,2\} . R$ is a finite commutative ring with 27 elements. This ring is a semi local ring with three maximal ideals. $R$ is a principal ideal ring and not finite chain ring. The units of the ring are $1,2,1+v^{2}, 1+v+2 v^{2}, 1+2 v+2 v^{2}, 2+v+$ $v^{2}, 2+2 v+v^{2}, 2+2 v^{2}$. The maximal ideals,

$$
\begin{aligned}
\langle v\rangle= & \langle 2 v\rangle=\left\langle v^{2}\right\rangle=\left\langle 2 v^{2}\right\rangle \\
= & \left\{0, v, 2 v, v^{2}, 2 v^{2}, v+v^{2}, v+2 v^{2}, 2 v+v^{2},\right. \\
& \left.2 v+2 v^{2}\right\} \\
\langle 1+v\rangle= & \langle 2+2 v\rangle=\left\langle 1+2 v+v^{2}\right\rangle=\left\langle 2+v+2 v^{2}\right\rangle \\
= & \left\{0,1+v, 2+2 v, v+v^{2}, 2 v+2 v^{2}, 1+2 v\right. \\
& \left.+v^{2}, 1+2 v^{2}, 2+v^{2}, 2+v+2 v^{2}\right\} \\
\left\langle 1+v+v^{2}\right\rangle= & \langle 1+2 v\rangle=\langle 2+v\rangle=\left\langle 2+2 v+2 v^{2}\right\rangle \\
= & \left\{0,2+v, 1+2 v, 2 v+v^{2}, v+2 v^{2}, 2+v^{2},\right. \\
& \left.1+2 v^{2}, 2+2 v+2 v^{2}, 1+v+v^{2}\right\}
\end{aligned}
$$


The other ideals,

$$
\begin{aligned}
\langle 0\rangle & =\{0\} \\
\langle 1\rangle & =\langle 2\rangle=\left\langle 1+v^{2}\right\rangle=\left\langle 1+v+2 v^{2}\right\rangle \\
& =\left\langle 1+2 v+2 v^{2}\right\rangle=\left\langle 2+v+v^{2}\right\rangle \\
& =\left\langle 2+2 v+v^{2}\right\rangle=\left\langle 2+2 v^{2}\right\rangle=R \\
\left\langle 1+2 v^{2}\right\rangle & =\left\langle 2+v^{2}\right\rangle=\left\{0,2+v^{2}, 1+2 v^{2}\right\} \\
\left\langle v+v^{2}\right\rangle & =\left\langle 2 v+2 v^{2}\right\rangle=\left\{0, v+v^{2}, 2 v+2 v^{2}\right\} \\
\left\langle v+2 v^{2}\right\rangle & =\left\langle 2 v+v^{2}\right\rangle=\left\{0, v+2 v^{2}, 2 v+v^{2}\right\}
\end{aligned}
$$

A linear code $C$ over $R$ length $n$ is a $R$-submodule of $R^{n}$. An element of $C$ is called a codeword.

For any $x=\left(x_{0}, x_{1}, \ldots, x_{n-1}\right), y=\left(y_{0}, y_{1}, \ldots, y_{n-1}\right)$ the inner product is defined as

$$
x . y=\sum_{i=0}^{n-1} x_{i} y_{i}
$$

If $x . y=0$ then $x$ and $y$ are said to be orthogonal. Let $C$ be linear code of length $n$ over $R$, the dual code of $C$

$$
C^{\perp}=\{x: \forall y \in C, x . y=0\}
$$

which is also a linear code over $R$ of length $n$. A code $C$ is self orthogonal if $C \subseteq C^{\perp}$ and self dual if $C=C^{\perp}$.

A cyclic code $C$ over $R$ is a linear code with the property that if $c=\left(c_{0}, c_{1}, \ldots, c_{n-1}\right) \in C$ then $\sigma(C)=$ $\left(c_{n-1}, c_{0}, \ldots, c_{n-2}\right) \in C$. A subset $C$ of $R^{n}$ is a linear cyclic code of length $n$ iff it is polynomial representation is an ideal of $R[x] /\left\langle x^{n}-1\right\rangle$.

A constacyclic code $C$ over $R$ is a linear code with the property that if $c=\left(c_{0}, c_{1}, \ldots, c_{n-1}\right) \in C$ then $\nu(C)=$ $\left(\lambda c_{n-1}, c_{0}, \ldots, c_{n-2}\right) \in C$ where $\lambda$ is a unit element of $R$. A subset $C$ of $R^{n}$ is a linear $\lambda$-constacyclic code of length $n$ iff it is polynomial representation is an ideal of $R[x] /\left\langle x^{n}-\lambda\right\rangle$.

A negacyclic code $C$ over $R$ is a linear code with the property that if $c=\left(c_{0}, c_{1}, \ldots, c_{n-1}\right) \in C$ then $\eta(C)=$ $\left(-c_{n-1}, c_{0}, \ldots, c_{n-2}\right) \in C$. A subset $C$ of $R^{n}$ is a linear negacyclic code of length $n$ iff it is polynomial representation is an ideal of $R[x] /\left\langle x^{n}+1\right\rangle$.

Let $C$ be code over $Z_{3}$ of length $n$ and $\dot{c}=$ $\left(c_{0}, \dot{c}_{1}, \ldots, \dot{c}_{n-1}\right)$ be a codeword of $C$. The Hamming weight of $\dot{c}$ is defined as $w_{H}(\dot{c})=\sum_{i=0}^{n-1} w_{H}\left(\dot{c}_{i}\right)$ where $w_{H}\left(\dot{c}_{i}\right)=1$ if $\dot{c}_{i} \neq 0$ and $w_{H}\left(\dot{c}_{i}\right)=0$ if $\dot{c}_{i}=0$. Hamming distance of $C$ is defined as $d_{H}(C)=\min d_{H}(c, \dot{c})$, where for any $\dot{c} \in C, c \neq \dot{c}$ and $d_{H}(c, \dot{c})$ is Hamming distance between two codewords with $d_{H}(c, \dot{c})=w_{H}(c-\dot{c})$.

Let $a \in Z_{3}^{3 n}$ with $a=\left(a_{0}, a_{1}, \ldots, a_{3 n-1}\right)=$ $\left(a^{(0)}\left|a^{(1)}\right| a^{(2)}\right), \quad a^{(i)} \in Z_{3}^{n}$ for $i=0,1,2$. Let $\varphi$ be a map from $Z_{3}^{3 n}$ to $Z_{3}^{3 n}$ given by $\varphi(a)=\left(\sigma\left(a^{(0)}\right)\left|\sigma\left(a^{(1)}\right)\right| \sigma\left(a^{(2)}\right)\right)^{3}$ where $\sigma$ is a cyclic shift from $Z_{3}^{n}$ to $Z_{3}^{n}$ given by $\sigma\left(a^{(i)}\right)=\left(\left(a^{(i, n-1)}\right),\left(a^{(i, 0)}\right),\left(a^{(i, 1)}\right), \ldots,\left(a^{(i, n-2)}\right)\right)$ for every $a^{(i)}=\left(a^{(i, 0)}, \ldots, a^{(i, n-1)}\right)$ where $a^{(i, j)} \in Z_{3}$, $j=0,1, \ldots, n-1$. A code of length $3 n$ over $Z_{3}$ is said to be quasi cyclic code of index 3 if $\varphi(C)=C$.
Let $n=s l$. A quasi-cyclic code $C$ over $R$ of length $n$ and index $l$ is a linear code with the property that if

$e=\left(e_{0,0}, \ldots, e_{0, l-1}, e_{1,0}, \ldots, e_{1, l-1}, \ldots, e_{s-1,0}, \ldots, e_{s-1, l-1}\right) \in$ $C$, then $\tau_{s, l}(e)=\left(e_{s-1,0}, \ldots, e_{s-1, l-1}, e_{0,0}, \ldots, e_{0, l-1}, \ldots, e_{s-2,0}\right.$ $\left.\ldots, e_{s-2, l-1}\right) \in C$.

Let $a \in Z_{3}^{3 n}$ with $a=\left(a_{0}, a_{1}, \ldots, a_{3 n-1}\right)=$ $\left(a^{(0)}\left|a^{(1)}\right| a^{(2)}\right), a^{(i)} \in Z_{3}^{n}$, for $i=0,1,2$. Let $\Gamma$ be a map from $Z_{3}^{3 n}$ to $Z_{3}^{3 n}$ given by

$$
\Gamma(a)=\left(\mu\left(a^{(0)}\right)\left|\mu\left(a^{(1)}\right)\right| \mu\left(a^{(2)}\right)\right)
$$

where $\mu$ is the map from $Z_{3}^{n}$ to $Z_{3}^{n}$ given by

$$
\mu\left(a^{(i)}\right)=\left(\left(a^{(i, s-1)}\right),\left(a^{(i, 0)}\right), \ldots,\left(a^{(i, s-2)}\right)\right)
$$

for every $a^{(i)}=\left(a^{(i, 0)}, \ldots, a^{(i, s-1)}\right)$ where $a^{(i, j)} \in Z_{3}^{l}, j=$ $0,1, \ldots, s-1$ and $n=s l$. A code of length $3 n$ over $Z_{3}$ is said to be $l$-quasi cyclic code of index 3 if $\Gamma(C)=C$.

\section{GRAY MAP AND GRAY IMAGES OF CYCLIC AND QUASI-CYCLIC CODES OVER $R$}

In [4], the Gray map is defined as follows

$$
\begin{aligned}
& \phi \quad: \quad R \rightarrow Z_{3}^{3} \\
& \phi\left(a+v b+v^{2} c\right) \quad=\quad(a, a+b+c, a+2 b+c)
\end{aligned}
$$

Let $C$ be a linear code over $R$ of length $n$. For any codeword $c=\left(c_{0}, \ldots, c_{n-1}\right)$ the Lee weight of $c$ is defined as $w_{L}(c)=\sum_{i=0}^{n-1} w_{L}\left(c_{i}\right)$ and the Lee distance of $C$ is defined as $d_{L}(C)=\min d_{L}(c, \dot{c})$, where for any $\dot{c} \in C$, $c \neq \dot{c}$ and $d_{L}(c, \dot{c})$ is Lee distance between two codewords with $d_{L}(c, \dot{c})=w_{L}(c-\dot{c})$. Gray map $\phi$ can be extended to map from $R^{n}$ to $Z_{3}^{3 n}$.

Theorem 1: The Gray map $\phi$ is a weight preserving map from $\left(R^{n}\right.$, Lee weight $)$ to $\left(Z_{3}^{3 n}\right.$, Hamming weight $)$. Moreover it is an isometry from $R^{n}$ to $Z_{3}^{3 n}$.

Theorem 2: If $C$ is an $\left[n, k, d_{L}\right]$ linear codes over $R$ then $\phi(C)$ is a $\left[3 n, k, d_{H}\right]$ linear codes over $Z_{3}$, where $d_{H}=d_{L}$.

Proof: Let $x=a_{1}+v b_{1}+v^{2} c_{1}, y=a_{2}+v b_{2}+v^{2} c_{2} \in$ $R, \alpha \in Z_{3}$ then

$$
\begin{gathered}
\phi(x+y)=\phi\left(a_{1}+a_{2}+v\left(b_{1}+b_{2}\right)+v^{2}\left(c_{1}+c_{2}\right)\right) \\
=\left(a_{1}+a_{2}, a_{1}+a_{2}+b_{1}+b_{2}+c_{1}+c_{2}, a_{1}+a_{2}+\right. \\
\left.2\left(b_{1}+b_{2}\right)+c_{1}+c_{2}\right) \\
=\left(a_{1}, a_{1}+b_{1}+c_{1}, a_{1}+2 b_{1}+c_{1}\right)+\left(a_{2}, a_{2}+b_{2}+\right. \\
\left.c_{2}, a_{2}+2 b_{2}+c_{2}\right) \\
=\phi(x)+\phi(y) \\
\phi(\alpha x)=\phi\left(\alpha a_{1}+v \alpha b_{1}+v^{2} \alpha c_{1}\right) \\
=\left(\alpha a_{1}, \alpha a_{1}+\alpha b_{1}+\alpha c_{1}, \alpha a_{1}+2 \alpha b_{1}+\alpha c_{1}\right) \\
=\alpha\left(a_{1}, a_{1}+b_{1}+c_{1}, a_{1}+2 b_{1}+c_{1}\right) \\
=\alpha \phi(x)
\end{gathered}
$$

so $\phi$ is linear. As $\phi$ is bijective then $|C|=|\phi(C)|$. From Theorem 1 we have $d_{H}=d_{L}$.

Theorem 3: If $C$ is self orthogonal, so is $\phi(C)$. 
Proof: Let $x=a_{1}+v b_{1}+v^{2} c_{1}, y=a_{2}+v b_{2}+v^{2} c_{2}$ where $a_{1}, b_{1}, c_{1}, a_{2}, b_{2}, c_{2} \in Z_{3}$. From

$x . y=a_{1} a_{2}+v\left(a_{1} b_{2}+b_{1} a_{2}+b_{1} c_{2}+c_{1} b_{2}\right)+v^{2}\left(a_{1} c_{2}+\right.$ $\left.b_{1} b_{2}+c_{1} a_{2}+c_{1} c_{2}\right)$ if $C$ is self orthogonal, so we have

$$
\begin{gathered}
a_{1} a_{2}=0, \\
a_{1} b_{2}+b_{1} a_{2}+b_{1} c_{2}+c_{1} b_{2}=0, \\
a_{1} c_{2}+b_{1} b_{2}+c_{1} a_{2}+c_{1} c_{2}=0 .
\end{gathered}
$$

From

$\phi(x) . \phi(y)=\left(a_{1}, a_{1}+b_{1}+c_{1}, a_{1}+2 b_{1}+c_{1}\right)\left(a_{2}, a_{2}+b_{2}+\right.$ $\left.c_{2}, a_{2}+2 b_{2}+c_{2}\right)=a_{1} a_{2}+a_{1} a_{2}+a_{1} b_{2}+a_{1} c_{2}+b_{1} a_{2}+b_{1} b_{2}+$ $b_{1} c_{2}+c_{1} a_{2}+c_{1} b_{2}+c_{1} c_{2}+a_{1} a_{2}+2\left(a_{1} b_{2}+b_{1} a_{2}+b_{1} c_{2}+\right.$ $\left.c_{1} b_{2}\right)+a_{1} c_{2}+b_{1} b_{2}+c_{1} a_{2}+c_{1} c_{2}=0$

Therefore, we have $\phi(C)$ is self orthogonal.

Note that $\phi(C)^{\perp}=\phi\left(C^{\perp}\right)$. Moreover, if $C$ is self-dual, so is $\phi(C)$.

Proposition 4: Let $\phi$ the Gray map from $R^{n}$ to $Z_{3}^{3 n}$, let $\sigma$ be cyclic shift and let $\varphi$ be a map as in the preliminaries. Then $\phi \sigma=\varphi \phi$.

Proof: Let $r_{i}=a_{i}+v b_{i}+v^{2} c_{i}$ be the elements of $R$ for $i=0,1, \ldots, n-1$. We have $\sigma\left(r_{0}, r_{1}, \ldots, r_{n-1}\right)=$ $\left(r_{n-1}, r_{0}, \ldots, r_{n-2}\right)$. If we apply $\phi$, we have

$$
\begin{aligned}
\phi\left(\sigma\left(r_{0}, \ldots, r_{n-1}\right)\right)= & \phi\left(r_{n-1}, r_{0}, \ldots, r_{n-2}\right) \\
= & \left(a_{n-1}, \ldots, a_{n-2}, a_{n-1}+b_{n-1}+c_{n-1}\right. \\
& , \ldots, a_{n-2}+b_{n-2}+c_{n-2}, a_{n-1}+ \\
& 2 b_{n-1}+c_{n-1}, \ldots, a_{n-2}+2 b_{n-2}+ \\
& \left.c_{n-2}\right)
\end{aligned}
$$

On the other hand $\phi\left(r_{0}, \ldots, r_{n-1}\right)=\left(a_{0}, \ldots, a_{n-1}, a_{0}+b_{0}+\right.$ $c_{0}, \ldots, a_{n-1}+b_{n-1}+c_{n-1}, a_{0}+2 b_{0}+c_{0}, \ldots, a_{n-1}+2 b_{n-1}+$ $\left.c_{n-1}\right)$. If we apply $\varphi$, we have $\varphi\left(\phi\left(r_{0}, r_{1}, \ldots, r_{n-1}\right)\right)=$ $\left(a_{n-1}, \ldots, a_{n-2}, a_{n-1}+b_{n-1}+c_{n-1}, \ldots, a_{n-2}+b_{n-2}+\right.$ $\left.c_{n-2}, a_{n-1}+2 b_{n-1}+c_{n-1}, \ldots, a_{n-2}+2 b_{n-2}+c_{n-2}\right)$. Thus, $\phi \sigma=\varphi \phi$.

Proposition 5: Let $\sigma$ and $\varphi$ be as in the preliminaries. A code $C$ of length $n$ over $R$ is cyclic code if and only if $\phi(C)$ is quasi cyclic code of index 3 over $Z_{3}$ with length $3 n$.

Proof: Suppose $C$ is cyclic code. Then $\sigma(C)=C$. If we apply $\phi$, we have $\phi(\sigma(C))=\phi(C)$. From Proposition $4, \phi(\sigma(C))=\varphi(\phi(C))=\phi(C)$. Hence, $\phi(C)$ is a quasi cyclic code of index 3 . Conversely, if $\phi(C)$ is a quasi cyclic code of index 3, then $\varphi(\phi(C))=\phi(C)$. From Proposition 4, we have $\varphi(\phi(C))=\phi(\sigma(C))=\phi(C)$. Since $\phi$ is injective, it follows that $\sigma(C)=C$.

Proposition 6: Let $\tau_{s, l}$ be quasi-cyclic shift on $R$. Let $\Gamma$ be as in the preliminaries. Then $\phi \tau_{s, l}=\Gamma \phi$.

Proof: Let $e=\left(e_{0,0}, \ldots, e_{0, l-1}, e_{1,0}, \ldots, e_{1, l-1}, \ldots, e_{s-1,0}\right.$, $\left.\ldots, e_{s-1, l-1}\right)$ with $e_{i, j}=a_{i, j}+v b_{i, j}+v^{2} c_{i, j}$ where $i \stackrel{=}{=}$ $0,1, \ldots, s-1$ and $j=0,1, \ldots, l-1$. We have $\tau_{s, l}(e)=$ $\left(e_{s-1,0}, \ldots, e_{s-1, l-1}, e_{0,0}, \ldots, e_{0, l-1}, \ldots, e_{s-2,0}, \ldots, e_{s-2, l-1}\right)$. If we apply $\phi$, we have

$$
\begin{aligned}
\phi\left(\tau_{s, l}(e)\right)= & \left(a_{s-1,0}, \ldots, a_{s-2, l-1}, a_{s-1,0}+b_{s-1,0}+c_{s-1,0}\right. \\
& , \ldots, a_{s-2, l-1}+b_{s-2, l-1}+c_{s-2, l-1}, a_{s-1,0}+ \\
& 2 b_{s-1,0}+c_{s-1,0}, \ldots, a_{s-2, l-1}+2 b_{s-2, l-1}+ \\
& \left.c_{s-2, l-1}\right)
\end{aligned}
$$

On the other hand,

$$
\begin{aligned}
\phi(e)= & \left(a_{0,0}, \ldots, a_{s-1, l-1}, a_{0,0}+b_{0,0}+c_{0,0}, \ldots, a_{s-1, l-1}+\right. \\
& b_{s-1, l-1}+c_{s-1, l-1}, a_{0,0}+2 b_{0,0}+c_{0,0}, \ldots, a_{s-1, l-1} \\
& \left.+2 b_{s-1, l-1}+c_{s-1, l-1}\right)
\end{aligned}
$$

$\Gamma(\varphi(e))=\left(a_{s-1,0}, \ldots, a_{s-2, l-1}, a_{s-1,0}+b_{s-1,0}+\right.$ $c_{s-1,0}, \ldots, a_{s-2, l-1}+b_{s-2, l-1}+c_{s-2, l-1}, a_{s-1,0}+2 b_{s-1,0}+$ $\left.c_{s-1,0}, \ldots, a_{s-2, l-1}+2 b_{s-2, l-1}+c_{s-2, l-1}\right)$. So, we have $\varphi \tau_{s, l}=\Gamma \varphi$.

Theorem 7: The Gray image of a quasi-cyclic code over $R$ of length $n$ with index $l$ is a $l$-quasi cyclic code of index 3 over $Z_{3}$ with length $3 n$.

Proof: Let $C$ be a quasi-cyclic code over $R$ of length $n$ with index $l$. That is $\tau_{s, l}(C)=C$. If we apply $\phi$, we have $\phi\left(\tau_{s, l}(C)\right)=\phi(C)$. From the Proposition 6, $\phi\left(\tau_{s, l}(C)\right)=$ $\phi(C)=\Gamma(\phi(C))$. So, $\phi(C)$ is a $l$ quasi-cyclic code of index 3 over $Z_{3}$ with length $3 n$.

We denote that $A_{1} \otimes A_{2} \otimes A_{3}=\left\{\left(a_{1}, a_{2}, a_{3}\right): a_{1} \in\right.$ $\left.A_{1}, a_{2} \in A_{2}, a_{3} \in A_{3}\right\}$ and $A_{1} \oplus A_{2} \oplus A_{3}=\left\{a_{1}+a_{2}+a_{3}\right.$ $\left.a_{1} \in A_{1}, a_{2} \in A_{2}, a_{3} \in A_{3}\right\}$

Let $C$ be a linear code of length $n$ over $R$. Define

$$
\begin{aligned}
& C_{1}=\left\{a \in Z_{3}^{n}: \exists b, c \in Z_{3}^{n}, a+v b+v^{2} c \in C\right\} \\
& C_{2}=\left\{a+b+c \in Z_{3}^{n}: a+v b+v^{2} c \in C\right\} \\
& C_{3}=\left\{a+2 b+c \in Z_{3}^{n}: a+v b+v^{2} c \in C\right\}
\end{aligned}
$$

Then $C_{1}, C_{2}$ and $C_{3}$ are ternary linear codes of length $n$. Moreover, the linear code $C$ of length $n$ over $R$ can be uniquely expressed as $C=\left(1+2 v^{2}\right) C_{1} \oplus\left(2 v+2 v^{2}\right) C_{2} \oplus$ $\left(v+2 v^{2}\right) C_{3}$.

Theorem 8: Let $C$ be a linear code of length $n$ over $R$. Then $\phi(C)=C_{1} \otimes C_{2} \otimes C_{3}$ and $|C|=\left|C_{1}\right|\left|C_{2}\right|\left|C_{3}\right|$.

Proof: For any $\left(a_{0}, a_{1}, \ldots, a_{n-1}, a_{0}+b_{0}+c_{0}, a_{1}+\right.$ $b_{1}+c_{1}, \ldots, a_{n-1}+b_{n-1}+c_{n-1}, a_{0}+2 b_{0}+c_{0}, a_{1}+2 b_{1}+$ $\left.c_{1}, \ldots, a_{n-1}+2 b_{n-1}+c_{n-1}\right) \in \phi(C)$. Let $m_{i}=a_{i}+$ $v b_{i}+v^{2} c_{i}, i=0,1, \ldots, n-1$. Since $\phi$ is a bijection $m=$ $\left(m_{0}, m_{1}, \ldots, m_{n-1}\right) \in C$. By definitions of $C_{1}, C_{2}$ and $C_{3}$ we have $\left(a_{0}, a_{1}, \ldots, a_{n-1}\right) \in C_{1},\left(a_{0}+b_{0}+c_{0}, a_{1}+b_{1}+\right.$ $\left.c_{1}, \ldots, a_{n-1}+b_{n-1}+c_{n-1}\right) \in C_{2},\left(a_{0}+2 b_{0}+c_{0}, a_{1}+2 b_{1}+\right.$ $\left.c_{1}, \ldots, a_{n-1}+2 b_{n-1}+c_{n-1}\right) \in C_{3}$. So, $\left(a_{0}, a_{1}, \ldots, a_{n-1}, a_{0}+\right.$ $b_{0}+c_{0}, a_{1}+b_{1}+c_{1}, \ldots, a_{n-1}+b_{n-1}+c_{n-1}, a_{0}+2 b_{0}+c_{0}, a_{1}+$ $\left.2 b_{1}+c_{1}, \ldots, a_{n-1}+2 b_{n-1}+c_{n-1}\right) \in C_{1} \otimes C_{2} \otimes C_{3}$. That is $\phi(C) \subseteq C_{1} \otimes C_{2} \otimes C_{3}$.

On the other hand, for any $(a, b, c) \in C_{1} \otimes C_{2} \otimes C_{3}$ where $a=\left(a_{0}, a_{1}, \ldots, a_{n-1}\right) \in C_{1}, b=\left(a_{0}+b_{0}+\right.$ $\left.c_{0}, a_{1}+b_{1}+c_{1}, \ldots, a_{n-1}+b_{n-1}+c_{n-1}\right) \in C_{2}, c=\left(a_{0}+\right.$ $\left.2 b_{0}+c_{0}, a_{1}+2 b_{1}+c_{1}, \ldots, a_{n-1}+2 b_{n-1}+c_{n-1}\right) \in C_{3}$. There are $x=\left(x_{0}, x_{1}, \ldots, x_{n-1}\right), y=\left(y_{0}, y_{1}, \ldots, y_{n-1}\right)$, $z=\left(z_{0}, z_{1}, \ldots, z_{n-1}\right) \in C$ such that $x_{i}=a_{i}+\left(v+2 v^{2}\right) p_{i}$, $y_{i}=b_{i}+\left(1+2 v^{2}\right) q_{i}, z_{i}=c_{i}+\left(2 v+2 v^{2}\right) r_{i}$ where $p_{i}, q_{i}, r_{i} \in Z_{3}$ and $0 \leq i \leq n-1$. Since $C$ is linear we have $m=\left(1+2 v^{2}\right) x+\left(2 v+2 v^{2}\right) y+\left(v+2 v^{2}\right) z=$ $a+v(2 b+c)+v^{2}(2 a+2 b+2 c) \in C$. It follows then $\phi(m)=(a, b, c)$, which gives $C_{1} \otimes C_{2} \otimes C_{3} \subseteq \phi(C)$.

Therefore, $\phi(C)=C_{1} \otimes C_{2} \otimes C_{3}$. The second result is easy to verify. 
Corollary 9: If $\phi(C)=C_{1} \otimes C_{2} \otimes C_{3}$, then $C=(1+$ $\left.2 v^{2}\right) C_{1} \oplus\left(2 v+2 v^{2}\right) C_{2} \oplus\left(v+2 v^{2}\right) C_{3}$. It is easy to see that

$$
\begin{aligned}
|C| & =\left|C_{1}\right|\left|C_{2}\right|\left|C_{3}\right|=3^{n-\operatorname{deg}\left(f_{1}\right)} 3^{n-\operatorname{deg}\left(f_{2}\right)} 3^{n-\operatorname{deg}\left(f_{3}\right)} \\
& =3^{3 n-\left(\operatorname{deg}\left(f_{1}\right)+\operatorname{deg}\left(f_{2}\right)+\operatorname{deg}\left(f_{3}\right)\right)}
\end{aligned}
$$

where $f_{1}, f_{2}$ and $f_{3}$ are the generator polynomials of $C_{1}, C_{2}$ and $C_{3}$, respectively.

Corollary 10: If $G_{1}, G_{2}$ and $G_{3}$ are generator matrices of ternary linear codes $C_{1}, C_{2}$ and $C_{3}$ respectively, then the generator matrix of $C$ is

$$
G=\left[\begin{array}{c}
\left(1+2 v^{2}\right) G_{1} \\
\left(2 v+2 v^{2}\right) G_{2} \\
\left(v+2 v^{2}\right) G_{3}
\end{array}\right]
$$

We have

$$
\phi(G)=\left[\begin{array}{c}
\phi\left(\left(1+2 v^{2}\right) G_{1}\right) \\
\phi\left(\left(2 v+2 v^{2}\right) G_{2}\right) \\
\phi\left(\left(v+2 v^{2}\right) G_{3}\right)
\end{array}\right]=\left[\begin{array}{ccc}
G_{1} & 0 & 0 \\
0 & G_{2} & 0 \\
0 & 0 & G_{3}
\end{array}\right] .
$$

Let $d_{L}$ minimum Lee weight of linear code $C$ over $R$. Then, $d_{L}=d_{H}(\phi(C))=\min \left\{d_{H}\left(C_{1}\right), d_{H}\left(C_{2}\right), d_{H}\left(C_{3}\right)\right\}$ where $d_{H}\left(C_{i}\right)$ denotes the minimum Hamming weights of ternary codes $C_{1}, C_{2}$ and $C_{3}$, respectively.

As similiar to section 4 in [4] we have the following Lemma and Examples.

Lemma 11: Let $C=\langle f(x)\rangle$ be a negacyclic code of length $\mathrm{n}$ over $\mathrm{R}$ and $\phi(f(x))=\left(f_{1}, f_{2}, f_{3}\right)$ with $\operatorname{deg}\left(\operatorname{gcd}\left(f_{1}, x^{n}+\right.\right.$ 1) $)=n-k_{1}, \operatorname{deg}\left(\operatorname{gcd}\left(f_{2}, x^{n}+1\right)\right)=n-k_{2}, \operatorname{deg}\left(\operatorname{gcd}\left(f_{3}, x^{n}+\right.\right.$ 1) $=n-k_{3}$. Then, $|C|=3^{k_{1}+k_{2}+k_{3}}$.

Example 12: Let $C=\langle f(x)\rangle=\left\langle\left(2 v+2 v^{2}\right) x^{2}+(1+2 v+\right.$ $\left.\left.2 v^{2}\right) x+1\right\rangle$ be a negacyclic code of length 3 over $R$. Hence, $\phi(f(x))=\left(x+1, x^{2}+2 x+1, x+1\right)$ and

$$
\begin{aligned}
& f_{1}=\operatorname{gcd}\left(x+1, x^{3}+1\right)=x+1 \\
& f_{2}=\operatorname{gcd}\left(x^{2}+2 x+1, x^{3}+1\right)=x^{2}+2 x+1 \\
& f_{3}=\operatorname{gcd}\left(x+1, x^{3}+1\right)=x+1
\end{aligned}
$$

So we have $|C|=3^{2+1+2}=3^{5}$.

Example 13: Let $C=\langle f(x)\rangle=\left\langle v^{2} x^{4}+v x^{3}+(1+\right.$ $\left.\left.2 v^{2}\right) x^{2}+2 v x+1\right\rangle$ be a negacyclic code of length 10 over $R$. Hence, $\phi(f(x) t)=\left(x^{2}+1, x^{4}+x^{3}+2 x+1, x^{4}+2 x^{3}+x+1\right)$ and

$f_{1}=\operatorname{gcd}\left(x^{2}+1, x^{10}+1\right)=x^{2}+1$

$f_{2}=\operatorname{gcd}\left(x^{4}+x^{3}+2 x+1, x^{10}+1\right)=x^{4}+x^{3}+2 x+1$

$f_{3}=\operatorname{gcd}\left(x^{4}+2 x^{3}+x+1, x^{10}+1\right)=x^{4}+2 x^{3}+x+1$

So we have $|C|=3^{8+6+6}=3^{20}$.

Let $h_{i}(x)=\left(x^{n}+1\right) /\left(\operatorname{gcd}\left(x^{n}+1, f_{i}\right)\right)$. Hence, $C^{\perp}=$ $\left\langle\phi^{-1}\left(h_{1_{R}}(x), h_{2_{R}}(x), h_{3_{R}}(x)\right)\right\rangle$ where $h_{i_{R}}(x)$ be the reciprocal polynomial of $h_{i}(x)$ for $i=1,2,3$. By using the previous
Example 13,

$$
\begin{aligned}
C^{\perp}= & \left\langle\phi^{-1}\left(h_{1_{R}}(x), h_{2_{R}}(x), h_{3_{R}}(x)\right)\right\rangle \\
= & \left\langle\phi ^ { - 1 } \left( x^{8}+2 x^{6}+x^{4}+2 x^{2}+1, x^{6}+x^{5}+x^{4}+x^{2}\right.\right. \\
& \left.\left.+2 x+1, x^{6}+2 x^{5}+x^{4}+x^{2}+x+1\right)\right\rangle \\
= & \left\langle\left(1+2 v^{2}\right) x^{8}+\left(2+2 v^{2}\right) x^{6}+v x^{5}+x^{4}\right. \\
& \left.+\left(2+2 v^{2}\right) x^{2}+2 v x+1\right\rangle
\end{aligned}
$$

\section{Quantum CODES From Cyclic (Negacyclic) CODES OVER $R$}

Theorem 14: Let $C_{1}=\left[n, k_{1}, d_{1}\right]_{q}$ and $C_{2}=\left[n, k_{2}, d_{2}\right]_{q}$ be linear codes over $\mathrm{GF}(\mathrm{q})$ with $C_{2}^{\perp} \subseteq C_{1}$. Furthermore, let $d=\min \left\{w t(v): v \in\left(C_{1} \backslash C_{2}^{\perp}\right) \cup\left(C_{2}^{\perp} \backslash C_{1}\right)\right\} \geq$ $\min \left\{d_{1}, d_{2}\right\}$.Then there exists a quantum error-correcting code $C=\left[n, k_{1}+k_{2}-n, d\right]_{q}$.In particular, if $C_{1}^{\perp} \subseteq C_{1}$, then there exists a quantum error-correcting code $C=\left[n, n-2 k_{1}, d_{1}\right]$, where $d_{1}=\min \left\{w t(v): v \in\left(C_{1}^{\perp} \backslash C_{1}\right)\right\}[16]$.

Proposition 15: Let $C=\left(1+2 v^{2}\right) C_{1} \oplus\left(2 v+2 v^{2}\right) C_{2} \oplus$ $\left(v+2 v^{2}\right) C_{3}$ be a linear code over $R$. Then $C$ is a cyclic code over $R$ iff $C_{1}, C_{2}$ and $C_{3}$ are cyclic codes.

Proof: Let $\left(a_{0}, a_{1}, \ldots, a_{n-1}\right) \in C_{1},\left(b_{0}, b_{1}, \ldots, b_{n-1}\right) \in$ $C_{2}$ and $\left(c_{0}, c_{1}, \ldots, c_{n-1}\right) \in C_{3}$. Assume that $m_{i}=(1+$ $\left.2 v^{2}\right) a_{i}+\left(2 v+2 v^{2}\right) b_{i}+\left(v+2 v^{2}\right) c_{i}$ for $i=0,1, \ldots, n-$ 1. Then $\left(m_{0}, m_{1}, \ldots, m_{n-1}\right) \in C$. Since $C$ is a cyclic code, it follows that $\left(m_{n-1}, m_{0}, \ldots, m_{n-2}\right) \in C$. Note that $\left(m_{n-1}, m_{0}, \ldots, m_{n-2}\right)=\left(1+2 v^{2}\right)\left(a_{n-1}, a_{0}, \ldots, a_{n-2}\right)+(2 v+$ $\left.2 v^{2}\right)\left(b_{n-1}, b_{0}, \ldots, b_{n-2}\right)+\left(v+2 v^{2}\right)\left(c_{n-1}, c_{0}, \ldots, c_{n-2}\right)$. Hence $\left(a_{n-1}, a_{0}, \ldots, a_{n-2}\right) \in C_{1},\left(b_{n-1}, b_{0}, \ldots, b_{n-2}\right) \in C_{2}$ and $\left(c_{n-1}, c_{0}, \ldots, c_{n-2}\right) \in C_{3}$. Therefore, $C_{1}, C_{2}$ and $C_{3}$ cyclic codes over $Z_{3}$.

Conversely, suppose that $C_{1}, C_{2}$ and $C_{3}$ cyclic codes over $Z_{3}$. Let $\left(m_{0}, m_{1}, \ldots, m_{n-1}\right) \in C$ where $m_{i}=\left(1+2 v^{2}\right) a_{i}+\left(2 v+2 v^{2}\right) b_{i}+\left(v+2 v^{2}\right) c_{i}$ for $i=0,1, \ldots, n-1$. Then $\left(a_{0}, a_{1}, \ldots, a_{n-1}\right) \in$ $C_{1},\left(b_{0}, b_{1}, \ldots, b_{n-1}\right) \in C_{2}$ and $\left(c_{0}, c_{1}, \ldots, c_{n-1}\right) \in$ $C_{3}$. Note that $\left(m_{n-1}, m_{0}, \ldots, m_{n-2}\right)=(1+$ $\left.2 v^{2}\right)\left(a_{n-1}, a_{0}, \ldots, a_{n-2}\right)+\left(2 v+2 v^{2}\right)\left(b_{n-1}, b_{0}, \ldots, b_{n-2}\right)+$ $\left(v+2 v^{2}\right)\left(c_{n-1}, c_{0}, \ldots, c_{n-2}\right) \in C=\left(1+2 v^{2}\right) C_{1} \oplus$ $\left(2 v+2 v^{2}\right) C_{2} \oplus\left(v+2 v^{2}\right) C_{3}$. So, $C$ is cyclic code over $R$.

Proposition 16: Let $C=\left(1+2 v^{2}\right) C_{1} \oplus\left(2 v+2 v^{2}\right) C_{2} \oplus$ $\left(v+2 v^{2}\right) C_{3}$ be a linear code over $R$. Then $C$ is a negacyclic code over $R$ iff $C_{1}, C_{2}$ and $C_{3}$ are negacyclic codes.

Proof: Let $\left(a_{0}, a_{1}, \ldots, a_{n-1}\right) \in C_{1},\left(b_{0}, b_{1}, \ldots, b_{n-1}\right) \in$ $C_{2}$ and $\left(c_{0}, c_{1}, \ldots, c_{n-1}\right) \in C_{3}$. Assume that $m_{i}=\left(1+2 v^{2}\right) a_{i}+\left(2 v+2 v^{2}\right) b_{i}+\left(v+2 v^{2}\right) c_{i}$ for $i=0,1, \ldots, n-1$. Then $\left(m_{0}, m_{1}, \ldots, m_{n-1}\right) \in C$. Since $C$ is a negacyclic code, it follows that $\left(-m_{n-1}, m_{0}, \ldots, m_{n-2}\right) \in C$. Note that $\left(-m_{n-1}, m_{0}, \ldots, m_{n-2}\right)=(1+$ $\left.2 v^{2}\right)\left(-a_{n-1}, a_{0}, \ldots, a_{n-2}\right)+\left(2 v+2 v^{2}\right)\left(-b_{n-1}, b_{0}, \ldots, b_{n-2}\right)+$ $\left(v+2 v^{2}\right)\left(-c_{n-1}, c_{0}, \ldots, c_{n-2}\right)$. Hence $\left(-a_{n-1}, a_{0}, \ldots, a_{n-2}\right) \in$ $C_{1},\left(-b_{n-1}, b_{0}, \ldots, b_{n-2}\right) \in C_{2}$ and $\left(-c_{n-1}, c_{0}, \ldots, c_{n-2}\right) \in$ $C_{3}$. Therefore, $C_{1}, C_{2}$ and $C_{3}$ negacyclic codes over $Z_{3}$.

Conversely, suppose that $C_{1}, C_{2}$ and $C_{3}$ negacyclic codes over $Z_{3}$. Let $\left(m_{0}, m_{1}, \ldots, m_{n-1}\right) \in C$ where $m_{i}=\left(1+2 v^{2}\right) a_{i}+\left(2 v+2 v^{2}\right) b_{i}+\left(v+2 v^{2}\right) c_{i}$ for $i=0,1, \ldots, n-1$. Then $\left(a_{0}, a_{1}, \ldots, a_{n-1}\right) \in C_{1}$, 
$\left(b_{0}, b_{1}, \ldots, b_{n-1}\right) \in C_{2}$ and $\left(c_{0}, c_{1}, \ldots, c_{n-1}\right) \in C_{3}$ Note that $\left(-m_{n-1}, m_{0}, \ldots, m_{n-2}\right) \quad=\quad(1+$ $\left.2 v^{2}\right)\left(-a_{n-1}, a_{0}, \ldots, a_{n-2}\right)+\left(2 v+2 v^{2}\right)\left(-b_{n-1}, b_{0}, \ldots, b_{n-2}\right)+$ $\left(v+2 v^{2}\right)\left(-c_{n-1}, c_{0}, \ldots, c_{n-2}\right) \in C=\left(1+2 v^{2}\right) C_{1} \oplus(2 v+$ $\left.2 v^{2}\right) C_{2} \oplus\left(v+2 v^{2}\right) C_{3}$. So, $C$ is negacyclic code over $R$.

Proposition 17: Suppose $C=\left(1+2 v^{2}\right) C_{1} \oplus$ $\left(2 v+2 v^{2}\right) C_{2} \oplus\left(v+2 v^{2}\right) C_{3}$ is a cyclic (negacyclic) code of length $n$ over $R$. Then

$$
C=<\left(1+2 v^{2}\right) f_{1},\left(2 v+2 v^{2}\right) f_{2},\left(v+2 v^{2}\right) f_{3}>
$$

and $|C|=3^{3 n-\left(\operatorname{deg} f_{1}+\operatorname{deg} f_{2}+\operatorname{deg} f_{3}\right)}$ where $f_{1}, f_{2}$ and $f_{3}$ generator polynomials of $C_{1}, C_{2}$ and $C_{3}$ respectively.

Proposition 18: Suppose $C$ is a cyclic (negacyclic) code of length $n$ over $R$, then there is a unique polynomial $f(x)$ such that $C=\langle f(x)\rangle$ and $f(x) \mid x^{n}-1\left(f(x) \mid x^{n}+1\right)$ where $f(x)=\left(1+2 v^{2}\right) f_{1}(x)+\left(2 v+2 v^{2}\right) f_{2}(x)+\left(v+2 v^{2}\right) f_{3}(x)$.

Proposition 19: Let $C$ be a linear code of length $n$ over $R$, then $C^{\perp}=\left(1+2 v^{2}\right) C_{1}^{\perp} \oplus\left(2 v+2 v^{2}\right) C_{2}^{\perp} \oplus\left(v+2 v^{2}\right) C_{3}^{\perp}$. Furthermore, $C$ is self-dual code iff $C_{1}, C_{2}$ and $C_{3}$ are selfdual codes over $Z_{3}$.

Proposition 20: If $C=\left(1+2 v^{2}\right) C_{1} \oplus\left(2 v+2 v^{2}\right) C_{2} \oplus$ $\left(v+2 v^{2}\right) C_{3}$ is a cyclic (negacyclic) code of length $n$ over $R$. Then

$$
C^{\perp}=\left\langle\left(1+2 v^{2}\right) h_{1}^{*}+\left(2 v+2 v^{2}\right) h_{2}^{*}+\left(v+2 v^{2}\right) h_{3}^{*}\right\rangle
$$

and $\left|C^{\perp}\right|=3^{\operatorname{deg} f_{1}+\operatorname{deg} f_{2}+\operatorname{deg} f_{3}}$ where for $i=1,2,3$, $h_{i}^{*}$ are the reciprocal polynomials of $h_{i}$ i.e., $h_{i}(x)=$ $\left(x^{n}-1\right) / f_{i}(x),\left(h_{i}(x)=\left(x^{n}+1\right) / f_{i}(x)\right), h_{i}^{*}(x)=$ $x^{\operatorname{deg} h_{i}} h_{i}\left(x^{-1}\right)$ for $i=1,2,3$.

Lemma 21: A ternary linear cyclic (negacyclic) code $C$ with generator polynomial $f(x)$ contains its dual code iff

$$
x^{n}-1 \equiv 0\left(\bmod f f^{*}\right), \quad\left(x^{n}+1 \equiv 0\left(\bmod f f^{*}\right)\right)
$$

where $f^{*}$ is the reciprocal polynomial of $f$.

Theorem 22: Let $C=\left\langle\left(1+2 v^{2}\right) f_{1},\left(2 v+2 v^{2}\right) f_{2},(v+\right.$ $\left.\left.2 v^{2}\right) f_{3}\right\rangle$ be a cyclic (negacyclic ) code of length $n$ over $R$. Then $C^{\perp} \subseteq C$ iff $x^{n}-1 \equiv 0\left(\bmod f_{i} f_{i}^{*}\right)\left(x^{n}+1 \equiv 0\left(\bmod f_{i} f_{i}^{*}\right)\right)$ for $i=1,2,3$.

Proof: Let $x^{n}-1 \equiv 0\left(\bmod f_{i} f_{i}^{*}\right)\left(x^{n}+1 \equiv\right.$ $\left.0\left(\bmod f_{i} f_{i}^{*}\right)\right)$ for $i=1,2,3$. Then $C_{1}^{\perp} \subseteq C_{1}, C_{2}^{\perp} \subseteq$ $C_{2}, C_{3}^{\perp} \subseteq C_{3}$. By using $\left(1+2 v^{2}\right) C_{1}^{\bar{\perp}} \subseteq C^{\prime}(1+$ $\left.2 v^{2}\right) C_{1},\left(\overline{2 v}+2 v^{2}\right) C_{2}^{\perp} \subseteq\left(2 v+2 v^{2}\right) C_{2},\left(v+2 \bar{v}^{2}\right) C_{3}^{\perp} \subseteq$ $\left(v+2 v^{2}\right) C_{3}$. We have $\left(1+2 v^{2}\right) C_{1}^{\perp} \oplus\left(2 v+2 v^{2}\right) C_{2}^{\perp} \oplus$ $\left(v+2 v^{2}\right) C_{3}^{\perp} \subseteq\left(1+2 v^{2}\right) C_{1} \oplus\left(2 v+2 v^{2}\right) C_{2} \oplus\left(v+2 v^{2}\right) C_{3}$. So, $\left\langle\left(1+2 v^{2}\right) h_{1}^{*}+\left(2 v+2 v^{2}\right) h_{2}^{*}+\left(v+2 v^{2}\right) h_{3}^{*}\right\rangle \subseteq\langle(1+$ $\left.\left.2 v^{2}\right) f_{1},\left(2 v+2 v^{2}\right) f_{2},\left(v+2 v^{2}\right) f_{3}\right\rangle$. That is $C^{\perp} \subseteq \bar{C}$.

Conversely, if $C^{\perp} \subseteq C$, then $\left(1+2 v^{2}\right) C_{1}^{\perp} \oplus$ $\left(2 v+2 v^{2}\right) C_{2}^{\perp} \oplus\left(v+2 \bar{v}^{2}\right) C_{3}^{\perp} \subseteq\left(1+2 v^{2}\right) C_{1} \oplus$ $\left(2 v+2 v^{2}\right) C_{2} \oplus\left(v+2 v^{2}\right) C_{3}$. By thinking $\bmod (1+$ $\left.2 v^{2}\right), \bmod \left(2 v+2 v^{2}\right)$ and $\bmod \left(v+2 v^{2}\right)$ respectively we have $C_{i}^{\perp} \subseteq C_{i}$ for $i=1,2,3$. Therefore, $x^{n}-1 \equiv$ $0\left(\bmod f_{i} f_{i}^{*}\right)\left(x^{n}+1 \equiv 0\left(\bmod f_{i} f_{i}^{*}\right)\right)$ for $i=1,2,3$.

Corollary 23: $C=\left(1+2 v^{2}\right) C_{1} \oplus\left(2 v+2 v^{2}\right) C_{2} \oplus$ $\left(v+2 v^{2}\right) C_{3}$ is a cyclic (negacyclic) code of length $n$ over $R$. Then $C^{\perp} \subseteq C$ iff $C_{i}^{\perp} \subseteq C_{i}$ for $i=1,2,3$.
Example 24: Let $n=6, R=Z_{3}+v Z_{3}+v^{2} Z_{3}, v^{3}=v$. We have $x^{6}-1=\left(2 x^{2}+2\right)\left(x^{2}+2\right)\left(2 x^{2}+1\right)=f_{1} f_{2} f_{3}$ in $Z_{3}[x]$. Hence,

$$
\begin{aligned}
& f_{1}^{*}=2 x^{2}+2=f_{1} \\
& f_{2}^{*}=2 x^{2}+1=f_{3} \\
& f_{3}^{*}=x^{2}+2=f_{2}
\end{aligned}
$$

Let $C=\left\langle\left(1+2 v^{2}\right) f_{2},\left(2 v+2 v^{2}\right) f_{2},\left(v+2 v^{2}\right) f_{3}\right\rangle$. Obviously $x^{6}-1$ is divisibly by $f_{i} f_{i}^{*}$ for $i=2,3$. Thus we have $C^{\perp} \subseteq C$.

Example 25: Let $n=10, R=Z_{3}+v Z_{3}+v^{2} Z_{3}, v^{3}=v$. We have $x^{10}+1=\left(x^{2}+1\right)\left(x^{4}+x^{3}+2 x+1\right)\left(x^{4}+2 x^{3}+x+1\right)=$ $g_{1} g_{2} g_{3}$ in $Z_{3}[x]$. Hence,

$$
\begin{aligned}
& g_{1}^{*}=x^{2}+1=g_{1} \\
& g_{2}^{*}=x^{4}+2 x^{3}+x+1=g_{3} \\
& g_{3}^{*}=x^{4}+x^{3}+2 x+1=g_{2}
\end{aligned}
$$

Let $C=\left\langle\left(1+2 v^{2}\right) g_{2},\left(2 v+2 v^{2}\right) g_{2},\left(v+2 v^{2}\right) g_{3}\right\rangle$. Obviously $x^{10}+1$ is divisibly by $g_{i} g_{i}^{*}$ for $i=2,3$. Thus we have $C^{\perp} \subseteq C$.

Theorem 26: Let $C$ be linear code of length $n$ over $R$ with $|C|=3^{3 k_{1}+2 k_{2}+k_{3}}$ and minimum distance $d$. Then $\phi(C)$ is ternary linear $\left[3 n, 3 k_{1}+2 k_{2}+k_{3}, d\right]$ code.

Using Theorem 14 and Theorem 22 we can construct quantum codes.

Theorem 27: Let $\left(1+2 v^{2}\right) C_{1} \oplus\left(2 v+2 v^{2}\right) C_{2} \oplus$ $\left(v+2 v^{2}\right) C_{3}$ be a cyclic (negacyclic) code of arbitrary length $n$ over $R$ with type $27^{k_{1}} 9^{k_{2}} 3^{k_{3}}$. If $C_{i}^{\perp} \subseteq C_{i}$ where $i=1,2,3$ then $C^{\perp} \subseteq C$ and there exists a quantum error-correcting code with parameters $\left[\left[3 n, 2\left(3 k_{1}+2 k_{2}+k_{3}\right)-3 n, d_{L}\right]\right]$ where $d_{L}$ is the minimum Lee weights of $C$.

Example 28: Let $n=6$. We have $x^{6}-1=\left(2 x^{2}+2\right)\left(x^{2}+\right.$ 2) $\left(2 x^{2}+1\right)$ in $Z_{3}[x]$. Let $f_{1}(x)=f_{2}(x)=x^{2}+2, f_{3}=2 x^{2}+$ 1. Thus $C=<\left(1+2 v^{2}\right) f_{1},\left(2 v+2 v^{2}\right) f_{2},\left(v+2 v^{2}\right) f_{3}>$. $C$ is a linear cyclic code of length 6 .The dual code $C^{\perp}=$ $\left\langle\left(1+2 v^{2}\right) h_{1}^{*},\left(2 v+2 v^{2}\right) h_{2}^{*},\left(v+2 v^{2}\right) h_{3}^{*}\right\rangle$ can be obtained of Proposition 20. Clearly, $C^{\perp} \subseteq C$. Hence, we obtain a quantum code with parameters $[[18,6,2]]$.

Example 29: Let $n=8$. We have $x^{8}-1=(x+$ 1) $(x+2)\left(x^{2}+1\right)\left(x^{2}+x+2\right)\left(x^{2}+2 x+2\right)$ in $Z_{3}[x]$. Let $f_{1}(x)=f_{2}(x)=f_{3}(x)=x^{2}+1$. Thus $C=\langle(1+$ $\left.\left.2 v^{2}\right) f_{1},\left(2 v+2 v^{2}\right) f_{2},\left(v+2 v^{2}\right) f_{3}\right\rangle . C$ is a linear cyclic code of length 8 . Hence, we obtain a quantum code with parameters $[[24,12,2]]$.

Example 30: Let $n=12$. We have $x^{12}-1=$ $(x-1)^{3}\left(x^{3}+x^{2}+x+1\right)^{3}$ in $Z_{3}[x]$. Let $f_{1}(x)=f_{2}(x)=$ $f_{3}(x)=x^{3}+x^{2}+x+1$.Thus $C=\langle(1+$ $\left.\left.2 v^{2}\right) f_{1},\left(2 v+2 v^{2}\right) f_{2},\left(v+2 v^{2}\right) f_{3}\right\rangle . C$ is a linear cyclic code of length 12. The dual code $C^{\perp}=\left\langle\left(1+2 v^{2}\right) h_{1}^{*},(2 v+\right.$ $\left.\left.2 v^{2}\right) h_{2}^{*},\left(v+2 v^{2}\right) h_{3}^{*}\right\rangle$ can be obtained of Proposition 20. Clearly, $C^{\perp} \subseteq C$. Hence, we obtain a quantum code with parameters $[[3 \overline{6}, 18,2]]$.

Let $n=27$. We have $x^{27}-1=(x-1)^{3}\left(x^{3}-1\right)^{4}\left(x^{6}-\right.$ $\left.2 x^{3}+1\right)^{2}$ in $Z_{3}[x]$. Let $f_{1}(x)=f_{2}(x)=f_{3}(x)=x^{6}-2 x^{3}+1$. Hence, we obtain a quantum code with parameters $[[81,45,2]]$. 
Let $n=30$. We have $x^{30}-1=\left(x^{2}+2\right)^{3}\left(x^{4}+\right.$ $\left.x^{3}+x^{2}+x+1\right)^{3}\left(x^{4}+2 x^{3}+x^{2}+2 x+1\right)^{3}$ in $Z_{3}[x]$. Let $f_{1}(x)=f_{3}(x)=x^{4}+x^{3}+x^{2}+x+1$, $f_{2}(x)=x^{4}+2 x^{3}+x^{2}+2 x+1$. Hence, we obtain a quantum code with parameters $[[90,66,2]]$.

Example 31: Let $n=3$. We have $x^{3}+1=(x+1)^{3}$ in $Z_{3}[x]$. Let $f_{1}(x)=f_{2}(x)=f_{3}(x)=x+1$. Thus $C=\left\langle\left(1+2 v^{2}\right) f_{1},\left(2 v+2 v^{2}\right) f_{2},\left(v+2 v^{2}\right) f_{3}\right\rangle . C$ is a linear negacyclic code of length 3 . The dual code $C^{\perp}=<(1+$ $\left.2 v^{2}\right) h_{1}^{*},\left(2 v+2 v^{2}\right) h_{2}^{*},\left(v+2 v^{2}\right) h_{3}^{*}>$ can be obtained of Proposition 20. Clearly, $C^{\perp} \subseteq C$. Hence, we obtain a quantum code with parameters $[[9,3,2]]$.

Example 32: Let $n=10$. We have $x^{10}+1=\left(x^{2}+\right.$ 1) $\left(x^{4}+x^{3}+2 x+1\right)\left(x^{4}+2 x^{3}+x+1\right)$ in $Z_{3}[x]$. Let $f_{1}(x)=x^{4}+x^{3}+2 x+1, f_{2}(x)=f_{3}(x)=x^{4}+2 x^{3}+x+1$. Thus $C=\left\langle\left(1+2 v^{2}\right) f_{1},\left(2 v+2 v^{2}\right) f_{2},\left(v+2 v^{2}\right) f_{3}\right\rangle . C$ is a linear negacyclic code of length 10 . The dual code $C^{\perp}=$ $\left\langle\left(1+2 v^{2}\right) h_{1}^{*},\left(2 v+2 v^{2}\right) h_{2}^{*},\left(v+2 v^{2}\right) h_{3}^{*}\right\rangle$ can be obtained of Proposition 20. Clearly, $C^{\perp} \subseteq C$. Hence, we obtain a quantum code with parameters $[[30,6,4]]$.

Example 33: Let $n=12$. We have $x^{12}+1=\left(x^{4}+1\right)\left(x^{2}+\right.$ $x+2)\left(x^{2}+2 x+2\right)\left(2 x^{2}+2 x+1\left(2 x^{2}+x+1\right)\right.$ in $Z_{3}[x]$. Let $f_{1}(x)=x^{2}+x+2, f_{2}(x)=2 x^{2}+x+1, f_{3}(x)=x^{2}+2 x+2$. Thus $C=\left\langle\left(1+2 v^{2}\right) f_{1},\left(2 v+2 v^{2}\right) f_{2},\left(v+2 v^{2}\right) f_{3}\right\rangle . C$ is a linear negacyclic code of length 12 . The dual code $C^{\perp}=<$ $\left(1+2 v^{2}\right) h_{1}^{*},\left(2 v+2 v^{2}\right) h_{2}^{*},\left(v+2 v^{2}\right) h_{3}^{*}>$ can be obtained of Proposition 20. Clearly, $C^{\perp} \subseteq C$. Hence, we obtain a quantum code with parameters $[[36,2 \overline{4}, 2]]$.

\section{CONSTACYCLIC CODES OVER $R$}

Let $\lambda=\alpha+\beta v+\gamma v^{2}$ be unit element of $R$. Note that $\lambda^{n}=1$ if $n$ even $\lambda^{n}=\lambda$ if $n$ odd. So we only study $\lambda$ constacyclic codes of odd length.

Proposition 34: Let $\varrho$ be the map of $R[x] /\left\langle x^{n}-1\right\rangle$ into $R[x] /\left\langle x^{n}-\lambda\right\rangle$ defined by $\varrho(a(x))=a(\lambda x)$. If $n$ is odd, then $\varrho$ is a ring isomorphism.

Proof: The proof is straightforward if $n$ is odd, $a(x) \equiv b(x)\left(\bmod \left(x^{n}-1\right)\right)$ iff $a(\lambda x) \equiv b(\lambda x)\left(\bmod \left(x^{n}-\lambda\right)\right)$

Corollary 35: I is an ideal of $R[x] /\left\langle x^{n}-1\right\rangle$ if and only if $\varrho(I)$ is an ideal of $R[x] /\left\langle x^{n}-\lambda\right\rangle$.

Corollary 36: Let $\varrho$ be the permutation of $R^{n}$ with $\mathrm{n}$ odd, such that $\bar{\varrho}\left(a_{0}, a_{1}, \ldots, a_{n-1}\right)=\left(a_{0}, \lambda a_{1}, \lambda^{2} a_{2} \ldots, \lambda^{n-1} a_{n-1}\right)$ and $C$ be a subset of $R^{n}$ then $C$ is a linear cyclic code iff $\bar{\varrho}(C)$ is a linear $\lambda$-constacyclic code.

Corollary 37: $C$ is a cyclic code of parameters $\left(n, 3^{k}, d\right)$ over $R$ iff $\bar{\varrho}(C)$ is a $\lambda$-constacyclic code of parameters $\left(n, 3^{k}, d\right)$ over $R$, when $n$ is odd.

Theorem 38: Let $\lambda$ be a unit in $R$. Let $C=\left(1+2 v^{2}\right) C_{1} \oplus$ $\left(2 v+2 v^{2}\right) C_{2} \oplus\left(v+2 v^{2}\right) C_{3}$ be a linear code of length $n$ over $R$. Then $C$ is a $\lambda$-constacyclic code of length $n$ over $R$ iff $C_{i}$ are either cyclic codes or negacyclic codes of length $n$ over $Z_{3}$ for $i=1,2,3$.

Proof: Let $\nu$ be the $\lambda$-constacyclic shift on $R^{n}$. Let $C$ be a $\lambda$-constacyclic code of length $n$ over $R$.Let $\left(a_{0}, a_{1}, \ldots, a_{n-1}\right) \in C_{1},\left(b_{0}, b_{1}, \ldots, b_{n-1}\right) \in C_{2}$ and $\left(c_{0}, c_{1}, \ldots, c_{n-1}\right) \in C_{3}$. Then the corresponding element of $C$ is $\left(m_{0}, m_{1}, \ldots, m_{n-1}\right)=\left(1+2 v^{2}\right)\left(a_{0}, a_{1}, \ldots, a_{n-1}\right)+(2 v+$ $\left.2 v^{2}\right)\left(b_{0}, b_{1}, \ldots, b_{n-1}\right)+\left(v+2 v^{2}\right)\left(c_{0}, c_{1}, \ldots, c_{n-1}\right)$. Since $C$ is a $\lambda$-constacyclic code so, $\nu(m)=\left(\lambda m_{n-1}, m_{0}, \ldots, m_{n-2}\right) \in C$ where $m_{i}=a_{i}+b_{i} v+v^{2} c_{i}$ for $i=0,1, \ldots, n-1$. Let $\lambda=\alpha+v \beta+v^{2} \gamma$, where $\alpha, \beta, \gamma \in Z_{3} . \nu(m)=(1+$ $\left.2 v^{2}\right)\left(\lambda a_{n-1}, a_{0}, \ldots, a_{n-2}\right)+\left(2 v+2 v^{2}\right)\left(\lambda b_{n-1}, b_{0}, \ldots, b_{n-2}\right)+$ $\left(v+2 v^{2}\right)\left(\lambda c_{n-1}, c_{0}, \ldots, c_{n-2}\right)$. Since the units of $Z_{3}$ are 1 and -1 , so $\alpha=\overline{+} 1$. Therefore we have obtained the desired result. The other side it is seen easily.

\section{SKew CODES OVER $R$}

We are interested in studying skew codes using the ring $R=Z_{3}+v Z_{3}+v^{2} Z_{3}$ where $v^{3}=v$. We define non-trivial ring automorphism $\theta$ on the ring $R$ by $\theta\left(a+v b+v^{2} c\right)=$ $a+2 b v+v^{2} c$ for all $a+v b+v^{2} c \in R$.

The ring $R[x, \theta]=\left\{a_{0}+a_{1} x+\ldots+a_{n-1} x^{n-1}: a_{i} \in R\right.$, $n \in N\}$ is called a skew polynomial ring. This ring is a noncommutative ring. The addition in the ring $R[x, \theta]$ is the usual polynomial addition and multiplication is defined using the rule, $\left(a x^{i}\right)\left(b x^{j}\right)=a \theta^{i}(b) x^{i+j}$. Note that $\theta^{2}(a)=a$ for all $a \in R$. This implies that $\theta$ is a ring automorphism of order 2 .

Definition 39: A subset $C$ of $R^{n}$ is callled a skew cyclic code of length $n$ if $C$ satisfies the following conditions,

i) $C$ is a submodule of $R^{n}$,

ii) If $c=\left(c_{0}, c_{1}, \ldots, c_{n-1}\right) \in C$, then $\sigma_{\theta}(c)=$ $\left(\theta\left(c_{n-1)}, \theta\left(c_{0}\right), \ldots, \theta\left(c_{n-2}\right)\right) \in C\right.$.

Let $f(x)+\left(x^{n}-1\right)$ be an element in the set $R_{n}=$ $R[x, \theta] /\left(x^{n}-1\right)$ and let $r(x) \in R[x, \theta]$. Define multiplication from left as follows,

$$
r(x)\left(f(x)+\left(x^{n}-1\right)\right)=r(x) f(x)+\left(x^{n}-1\right)
$$

for any $r(x) \in R[x, \theta]$.

Theorem 40: $R_{n}$ is a left $R[x, \theta]$-module where multiplication defined as in above.

Theorem 41: A code $C$ in $R_{n}$ is a skew cyclic code if and only if $C$ is a left $R[x, \theta]$-submodule of the left $R[x, \theta]$ module $R_{n}$.

Theorem 42: Let $C$ be a skew cyclic code in $R_{n}$ and let $f(x)$ be a polynomial in $C$ of minimal degree. If $f(x)$ is monic polynomial, then $C=(f(x))$ where $f(x)$ is a right divisor of $x^{n}-1$.

Theorem 43: A module skew cyclic code of length $n$ over $R$ is free iff it is generated by a monic right divisor $f(x)$ of $x^{n}-1$. Moreover, the set $\left\{f(x), x f(x), x^{2} f(x), \ldots, x^{n-\operatorname{deg}(f(x))-1} f(x)\right\}$ forms a basis of $C$ and the rank of $C$ is $n-\operatorname{deg}(f(x))$.

Theorem 44: Let $n$ be odd and $C$ be a skew cyclic code of length $n$. Then $C$ is equivalent to cyclic code of length $n$ over $R$.

Proof: Since $n$ is odd, $\operatorname{gcd}(2, n)=1$. Hence there exist integers $b, c$ such that $2 b+n c=1$. So $2 b=1-n c=1+$ $z n$ where $z>0$. Let $a(x)=a_{0}+a_{1} x+\ldots+a_{n-1} x^{n-1}$ be a codeword in $C$. Note that $x^{2 b} a(x)=\theta^{2 b}\left(a_{0}\right) x^{1+z n}+$ 
$\theta^{2 b}\left(a_{1}\right) x^{2+z n}+\ldots+\theta^{2 b}\left(a_{n-1}\right) x^{n+z n}=a_{n-1}+a_{0} x+\ldots+$ $a_{n-2} x^{n-2} \in C$. Thus $C$ is a cyclic code of length $n$.

Corollary 45: Let $n$ be odd. Then the number of distinct skew cyclic codes of length $n$ over $R$ is equal to the number of ideals in $R[x] /\left(x^{n}-1\right)$ because of Theorem 44. If $x^{n}-1=$ $\prod_{i=0}^{r} p_{i}^{s_{i}}(x)$ where $p_{i}(x)$ are irreducible polynomials over $Z_{3}$. Then the number of distinct skew cyclic codes of length $n$ over $R$ is $\prod_{i=0}^{r}\left(s_{i}+1\right)^{3}$.

Example 46: Let $n=27$ and $f(x)=x^{3}-1$. Then $f(x)$ generates a skew cyclic codes of length 27. This code is equivalent to a cyclic code of length 27 . Since $x^{27}-1=$ $(x-1)^{3}\left(x^{3}-1\right)^{4}\left(x^{6}-2 x^{3}+1\right)^{2}$, it follows that there are $60^{3}$ skew cyclic code of length 27 .

Definition 47: A subset $C$ of $R^{n}$ is called a skew quasicyclic code of length $n$ if $C$ satisfies the following conditions, i) $C$ is a submodule of $R^{n}$,

ii) If $e=\left(e_{0,0}, \ldots, e_{0, l-1}, e_{1,0}, \ldots, e_{1, l-1}, \ldots, e_{s-1,0}, . ., e_{s-1, l-1}\right)$ $\in C$, then

$\tau_{\theta, s, l}(e)=\left(\theta\left(e_{s-1,0}\right), \ldots, \theta\left(e_{s-1, l-1}\right), \theta\left(e_{0,0}\right), \ldots, \theta\left(e_{0, l-1}\right), \ldots\right.$, $\left.\theta\left(e_{s-2,0}\right), \ldots, \theta\left(e_{s-2, l-1}\right)\right) \in C$.

We note that $x^{s}-1$ is a two sided ideal in $R[x, \theta]$ if $m \mid s$ where $m$ is the order of $\theta$ and equal to two. So $R[x, \theta] /\left(x^{s}-\right.$ $1)$ is well defined.

The ring $R_{s}^{l}=\left(R[x, \theta] /\left(x^{s}-1\right)\right)^{l}$ is a left $R_{s}=$ $R[x, \theta] /\left(x^{s}-1\right)$ module by the following multiplication on the left $f(x)\left(g_{1}(x), \ldots, g_{l}(x)\right)=\left(f(x) g_{1}(x), \ldots f(x) g_{l}(x)\right)$. If the map $\gamma$ is defined by

$$
\gamma: R^{n} \longrightarrow R_{s}^{l}
$$

$\left(e_{0,0}, \ldots, e_{0, l-1}, e_{1,0}, \ldots, e_{1, l-1}, \ldots, e_{s-1,0}, \ldots, e_{s-1, l-1}\right) \mapsto$ $\left(e_{0}(x), \ldots, e_{l-1}(x)\right)$ such that $e_{j}(x)=\sum_{i=0}^{s-1} e_{i, j} x^{i} \in R_{s}^{l}$ where $j=0,1, \ldots, l-1$ then the map $\gamma$ gives a one to one correspondence $R^{n}$ and the ring $R_{s}^{l}$.

Theorem 48: A subset $C$ of $R^{n}$ is a skew quasi-cyclic code of length $n=s l$ and index $l$ if and only if $\gamma(C)$ is a left $R_{s^{-}}$ submodule of $R_{s}^{l}$.

A code $C$ is said to be skew constacyclic if $C$ is closed the under the skew constacyclic shift $\sigma_{\theta, \lambda}$ from $R^{n}$ to $R^{n}$ defined by $\sigma_{\theta, \lambda}\left(\left(c_{0}, c_{1}, \ldots, c_{n-1}\right)\right)=$ $\left(\theta\left(\lambda c_{n-1}\right), \theta\left(c_{0}\right), \ldots, \theta\left(c_{n-2}\right)\right)$.

Privately, such codes are called skew cyclic and skew negacyclic codes when $\lambda$ is 1 and -1 , respectively.

Theorem 49: A code $C$ of length $n$ over $R$ is skew constacyclic iff the skew polynomial representation of $C$ is a left ideal in $R[x, \theta] /\left(x^{n}-\lambda\right)$.

\section{The Gray Images of Skew Codes Over $R$}

Proposition 50: Let $\sigma_{\theta}$ be the skew cyclic shift on $R^{n}$, let $\phi$ be the Gray map from $R^{n}$ to $Z_{3}^{3 n}$ and let $\varphi$ be as in the preliminaries. Then $\phi \sigma_{\theta}=\rho \varphi \phi$ where $\rho(x, y, z)=(x, z, y)$ for every $x, y, z \in Z_{3}^{n}$.

Proof: Let $r_{i}=a_{i}+v b_{i}+v^{2} c_{i}$ be the elements of $R$, for $i=0,1, \ldots, n-1$. We have $\sigma_{\theta}\left(r_{0}, r_{1}, \ldots, r_{n-1}\right)=$ $\left(\theta\left(r_{n-1}\right), \theta\left(r_{0}\right), \ldots, \theta\left(r_{n-2}\right)\right)$. If we apply $\phi$, we have

$$
\begin{aligned}
\phi\left(\sigma_{\theta}\left(r_{0}, \ldots, r_{n-1}\right)\right)= & \phi\left(\theta\left(r_{n-1}\right), \theta\left(r_{0}\right), \ldots, \theta\left(r_{n-2}\right)\right) \\
& \left(a_{n-1}, \ldots, a_{n-2}, a_{n-1}+2 b_{n-1}+\right. \\
& c_{n-1}, \ldots, a_{n-2}+2 b_{n-2}+c_{n-2}, \\
& a_{n-1}+b_{n-1}+c_{n-1}, \ldots, a_{n-2}+ \\
& \left.b_{n-2}+c_{n-2}\right)
\end{aligned}
$$

On the other hand, $\phi\left(r_{0}, \ldots, r_{n-1}\right)=\left(a_{0}, \ldots, a_{n-1}, a_{0}+b_{0}+\right.$ $c_{0}, \ldots, a_{n-1}+b_{n-1}+c_{n-1}, a_{0}+2 b_{0}+c_{0}, \ldots, a_{n-1}+2 b_{n-1}+$ $\left.c_{n-1}\right)$. If we apply $\varphi$, we have

$$
\begin{aligned}
& \varphi\left(\phi\left(r_{0}, r_{1}, \ldots, r_{n-1}\right)\right)=\left(a_{n-1}, \ldots, a_{n-2}, a_{n-1}+\right. \\
& b_{n-1}+c_{n-1}, \ldots, a_{n-2}+b_{n-2}+c_{n-2}, a_{n-1}+2 b_{n-1}+ \\
& \left.c_{n-1}, \ldots, a_{n-2}+2 b_{n-2}+c_{n-2}\right) . \text { If we apply } \rho \text {, we have } \\
& \rho\left(\varphi\left(\phi\left(r_{0}, r_{1}, \ldots, r_{n-1}\right)\right)=\left(a_{n-1}, \ldots, a_{n-2}, a_{n-1}+2 b_{n-1}+\right.\right. \\
& c_{n-1}, \ldots, a_{n-2}+2 b_{n-2}+c_{n-2}, a_{n-1}+b_{n-1}+c_{n-1}, \ldots, a_{n-2}+ \\
& \left.b_{n-2}+c_{n-2}\right) . \text { So, we have } \phi \sigma_{\theta}=\rho \varphi \phi .
\end{aligned}
$$

Theorem 51: The Gray image a skew cyclic code over $R$ of length $n$ is permutation equivalent to quasi-cyclic code of index 3 over $Z_{3}$ with length $3 n$.

Proof: Let $C$ be a skew cyclic codes over $S$ of length $n$. That is $\sigma_{\theta}(C)=C$. If we apply $\phi$, we have $\phi\left(\sigma_{\theta}(C)\right)=\phi(C)$. From the Proposition 50, $\phi\left(\sigma_{\theta}(C)\right)=\phi(C)=\rho(\varphi(\phi(C)))$. So, $\phi(C)$ is permutation equivalent to quasi-cyclic code of index 3 over $Z_{3}$ with length $3 n$.

Proposition 52: Let $\tau_{\theta, s, l}$ be skew quasi-cyclic shift on $R^{n}$, let $\phi$ be the Gray map from $R^{n}$ to $Z_{3}^{3 n}$, let $\Gamma$ be as in the preliminaries, let $\rho$ be as above. Then $\phi \tau_{\theta, s, l}=\rho \Gamma \phi$.

Theorem 53: The Gray image a skew quasi-cyclic code over $R$ of length $n$ with index $l$ is permutation equivalent to $l$ quasi-cyclic code of index 3 over $Z_{3}$ with length $3 n$.

Proposition 54: Let $\sigma_{\theta, \lambda}$ be skew constacyclic shift on $R^{n}$, let $\phi$ be the Gray map from $R^{n}$ to $Z_{3}^{3 n}$, let $\rho$ be as above. Then $\phi \nu=\rho \phi \sigma_{\theta, \lambda}$.

Theorem 55: The Gray image a skew constacyclic code over $R$ of length $n$ is permutation equivalent to the Gray image of a constacyclic code over $Z_{3}$ with length $3 n$.

The proofs of Proposition 52, 54 and Theorem 53, 55 are similiar to the proofs Proposition 50 and Theorem 51.

\section{QUASI-CONSTACYCLIC AND SKEW QUASI-CONSTACYCLIC CODES OVER $R$} $R$.

Let $M_{s}=R[x] /\left\langle x^{s}-\lambda\right\rangle$ where $\lambda$ is a unit element of

Definition 56: A subset $C$ of $R^{n}$ is a called a quasiconstacyclic code of length $n=l s$ with index $l$ if

i) $C$ is a submodule of $R^{n}$,

ii) if $e=\left(e_{0,0}, \ldots, e_{0, l-1}, e_{1,0}, \ldots, e_{1, l-1}, \ldots, e_{s-1,0}, \ldots, e_{s-1, l-1}\right)$ $\in C$ then

$\nabla_{\lambda, l}(e)=\left(\lambda e_{s-1,0}, \ldots, \lambda e_{s-1, l-1}, e_{0,0}, \ldots, e_{0, l-1}, e_{1,0}, \ldots, e_{1, l-1}\right.$ $\left., \ldots, e_{s-2,0}, \ldots, e_{s-2, l-1}\right) \in C$.

When $\lambda=1$ the quasi-constacyclic codes are just quasi-cyclic codes.

Since $x^{s}-\lambda=f_{1}(x) f_{2}(x) \ldots f_{r}(x)$, it follows that 


\section{$\left(R[x] /\left(x^{s}-\lambda\right)\right)^{l} \cong\left(R[x] /\left(f_{1}(x)\right)\right)^{l} \times\left(R[x] /\left(f_{2}(x)\right)\right)^{l} \times$ $\ldots \times\left(R[x] /\left(f_{r}(x)\right)\right)^{l}$.}

Every submodule of $\left(R[x] /\left(x^{s}-\lambda\right)\right)^{l}$ is a direct product of submodules of $\left(R[x] /\left(f_{t}(x)\right)\right)^{l}$ for $1 \leq t \leq r$.

Theorem 57: If $(s, 3)=1$ then a quasi-constacyclic code of length $n=s l$ with index $l$ over $R$ is a direct product of linear codes over $R[x] /\left(f_{t}(x)\right)$ for $1 \leq t \leq r$.

Let $x^{s}-\lambda=f_{1}(x) f_{2}(x) \ldots f_{r}(x)$ be the factorization of $x^{s}-\lambda$ into irreducible polynomials. Thus, if $(s, 3)=1$ and $C_{i}$ is a linear code of length $l$ over $R[x] /\left(f_{t}(x)\right)$ for $1 \leq t \leq r$, then $\prod_{t=1}^{r} C_{t}$ is a quasi-constacyclic code of length $n=s l$ over $R$ with $\prod_{t=1}^{r}\left|C_{t}\right|$ codewords.

Define a map $\chi: R^{n} \rightarrow M_{s}^{l}$ by $\chi(e)=$ $\left(e_{0}(x), e_{1}(x), \ldots, e_{l-1}(x)\right)$ where $e_{j}(x)=\sum_{i=o}^{s-1} e_{i j} x^{i} \in$ $M_{s}, j=0,1, \ldots, l-1$.

Lemma 58: Let $\chi(C)$ denote the image of $C$ under $\chi$. The map $\chi$ induces a one to one correspondence between quasiconstacyclic codes over $R$ of length $n$ with index $l$ and linear codes over $M_{s}$ of length $l$.

We define a conjugation map on $M_{s}$ as one that acts as the identity on the elements of $R$ and that sends $x$ to $x^{-1}=x^{s-1}$, and extended linearly.

We define on $R^{n=s l}$ the usual Euclidean inner product for

$$
e=\left(e_{0,0}, \ldots, e_{0, l-1}, e_{1,0}, \ldots, e_{1, l-1}, \ldots, e_{s-1,0}, \ldots, e_{s-1, l-1}\right)
$$

and

$$
c=\left(c_{0,0}, \ldots, c_{0, l-1}, c_{1,0}, \ldots, c_{1, l-1}, \ldots, c_{s-1,0}, \ldots, c_{s-1, l-1}\right)
$$

we define $e . c=\sum_{i=0}^{s-1} \sum_{j=0}^{l-1} e_{i j} c_{i j}$.

On $M_{s}^{l}$, we define the Hermitian inner product for $a(x)=\left(a_{0}(x), a_{1}(x), \ldots, a_{l-1}(x)\right)$ and $b(x)=$ $\left(b_{0}(x), b_{1}(x), \ldots, b_{l-1}(x)\right)$,

$$
\langle a, b\rangle=\sum_{j=0}^{l-1} a_{j}(x) \overline{b_{j}(x)} .
$$

Theorem 59: Let $e, c \in R^{n}$. Then $\left(\nabla_{\lambda, l}^{k}(e)\right) . c=0$ for all $k=0, \ldots, s-1$ iff $\langle\chi(e), \chi(c)\rangle=0$.

Corollary 60: Let $C$ be a quasi-constacyclic code of length $s l$ with index $l$ over $R$ and $\chi(C)$ be its image in $M_{s}^{l}$ under $\chi$. Then $\chi(C)^{\perp}=\chi\left(C^{\perp}\right)$, where the dual in $R^{s l}$ is taken with respect to the Euclidean inner product, while the dual in $M_{s}^{l}$ is taken with respect to the Hermitian inner product. The dual of a quasi-constacyclic code of length $s l$ with index $l$ over $R$ is a quasi-constacyclic code of length $s l$ with index $l$ over .

From [22] we get the following results.

Theorem 61: Let $\mathrm{C}$ be a quasi-constacyclic code of length $\mathrm{n}=\mathrm{sl}$ with index 1 over R. Let $C^{\perp}$ is the dual of C. If $C=$ $C_{1} \oplus C_{2} \oplus \ldots \oplus C_{r}$ then $C^{\perp}=C_{1}^{\perp} \oplus C_{2}^{\perp} \oplus \ldots \oplus C_{r}^{\perp}$.

Theorem 62: Let $C=C_{1} \oplus C_{2} \oplus \ldots \oplus C_{r}$ be a quasiconstacyclic code of length $\mathrm{n}=\mathrm{sl}$ with index 1 over $\mathrm{R}$ where $C_{t}$ is a free linear code of length 1 with rank $k_{t}$ over
$R[x] /\left(f_{t}(x)\right)$ for $1 \leq t \leq r$. Then $\mathrm{C}$ is a $\kappa$-generator quasiconstacyclic code and $C^{\perp}$ is an $\left(l-\kappa^{\prime}\right)$-generator quasiconstacyclic code where $\kappa=\max _{t}\left(k_{t}\right)$ and $\kappa^{\prime}=\min _{t}\left(k_{t}\right)$.

Let $M_{\theta, s}=R[x, \theta] /\left\langle x^{s}-\lambda\right\rangle$ where $\lambda$ is a unit element of $R$. Let $\theta$ be an automorphism of $R$ with $|\langle\theta\rangle|=m=2$.

Definition 63: A subset $C$ of $R^{n}$ is a called a skew quasi-constacyclic code of length $n=l s, m \mid s$, with index $l$ if i) $C$ is a submodule of $R^{n}$,

ii) if $e=\left(e_{0,0}, \ldots, e_{0, l-1}, e_{1,0}, \ldots, e_{1, l-1}, \ldots, e_{s-1,0}, \ldots, e_{s-1, l-1}\right)$ $\in C$ then

$\nabla_{\theta, \lambda, l}(e)=\left(\theta\left(\lambda e_{s-1,0}\right), \ldots, \theta\left(\lambda e_{s-1, l-1}\right), \theta\left(e_{0,0}\right), \ldots, \theta\left(e_{0, l-1}\right)\right.$ $\left., \theta\left(e_{1,0}\right), \ldots, \theta\left(e_{1, l-1}\right), \ldots, \theta\left(e_{s-2,0}\right), \ldots, \theta\left(e_{s-2, l-1}\right)\right) \in C$.

When $\lambda=1$ the skew quasi-constacyclic codes are just skew quasi-cyclic codes.

The ring $M_{\theta, s}^{l}$ is a left $M_{\theta, s}$ module where we define multiplication from left by $f(x)\left(g_{1}(x), \ldots, g_{l}(x)\right)=$ $\left(f(x) g_{1}(x), \ldots f(x) g_{l}(x)\right)$.

Define a map $\Lambda: R^{n} \rightarrow M_{\theta, s}^{l}$ by $\Lambda(e)=$ $\left(e_{0}(x), e_{1}(x), \ldots, e_{l-1}(x)\right)$ where $e_{j}(x)=\sum_{i=o}^{s-1} e_{i j} x^{i} \in$ $M_{\theta, s}, j=0,1, \ldots, l-1$.

Lemma 64: Let $\Lambda(C)$ denote the image of $C$ under $\Lambda$. The map $\Lambda$ induces a one to one correspondence between skew quasi-constacyclic codes over $R$ of length $n$ with index $l$ and linear codes over $M_{\theta, s}$ of length $l$.

Theorem 65: A subset $C$ of $R^{n}$ is a skew quasiconstacyclic code of length $n=l s$ with index $l$ iff is a left submodule of the ring $M_{\theta, s}^{l}$.

Proof: Let $C$ be a skew quasi-constacyclic code of index $l$ over R. Suppose that $\Lambda(C)$ forms a submodule of $M_{\theta, s}^{l}$. $\Lambda(C)$ is closed under addition and scalar multiplication. Let $\Lambda(e)=\left(e_{0}(x), e_{1}(x), \ldots, e_{l-1}(x)\right) \in \Lambda(C)$ for $e=$ $\left(e_{0,0}, \ldots, e_{0, l-1}, e_{1,0}, \ldots, e_{1, l-1}, \ldots, e_{s-1,0}, \ldots, e_{s-1, l-1}\right) \in C$. Then $x \Lambda(e) \in \Lambda(C)$. By linearity it follows that $r(x) \Lambda(e) \in$ $\Lambda(C)$ for any $r(x) \in M_{\theta, s}$. Therefore, $\Lambda(C)$ is a left module of $M_{\theta, s}^{l}$.

Conversely, suppose $E$ is an $M_{\theta, s}$ left submodule of $M_{\theta, s}^{l}$. Let $C=\Lambda^{-1}(E)=\left\{e \in R^{n}: \Lambda(e) \in E\right\}$. We claim that $C$ is a skew quasi-constacyclic code of $R$. Since $\Lambda$ is a isomorphism, $C$ is a linear code of length $n$ over $R$. Let $e=\left(e_{0,0}, \ldots, e_{0, l-1}, e_{1,0}, \ldots, e_{1, l-1}, \ldots, e_{s-1,0}, \ldots, e_{s-1, l-1}\right) \in$ $C$. Then $\Lambda(e)=\left(e_{0}(x), e_{1}(x), \ldots, e_{l-1}(x)\right) \in \Lambda(C)$, where $e_{j}(x)=\sum_{i=o}^{s-1} e_{i j} x^{i} \in M_{\theta, s}$ for $j=0,1, \ldots, l-1$. It is easy to see that $\bar{\Lambda}^{o}\left(\nabla_{\theta, \lambda, l}(e)\right)=x\left(e_{0}(x), e_{1}(x), \ldots, e_{l-1}(x)\right)=$ $\left(x e_{0}(x), x e_{1}(x), \ldots, x e_{l-1}(x)\right) \in E$. Hence $\nabla_{\theta, \lambda, l}(e) \in C$. So, $C$ is a skew quasi-constacyclic code $C$.

On $R^{n=s l}$ the usual Euclidean inner product for

$$
e=\left(e_{0,0}, \ldots, e_{0, l-1}, e_{1,0}, \ldots, e_{1, l-1}, \ldots, e_{s-1,0}, \ldots, e_{s-1, l-1}\right)
$$

and

$$
c=\left(c_{0,0}, \ldots, c_{0, l-1}, c_{1,0}, \ldots, c_{1, l-1}, \ldots, c_{s-1,0}, \ldots, c_{s-1, l-1}\right)
$$

we define $e . c=\sum_{i=0}^{s-1} \sum_{j=0}^{l-1} e_{i j} c_{i j}$. We define a conjugation map $\Omega$ on $M_{\theta, s}^{l}$ such that $\Omega\left(c x^{i}\right)=\theta^{-1}(c) x^{s-1}, 0 \leq$ $i \leq s-1$, and extended linearly. We define the Hermitian 
inner product for $a=\left(a_{0}(x), a_{1}(x), \ldots, a_{l-1}(x)\right)$ and $b=$ $\left(b_{0}(x), b_{1}(x), \ldots, b_{l-1}(x)\right)$,

$$
\langle a, b\rangle=\sum_{j=0}^{l-1} a_{j}(x) \Omega\left(b_{j}(x)\right) .
$$

Theorem 66: Let $e, c \in R^{n}$. Then $\left(\nabla_{\theta, \lambda, l}^{k}(e)\right) . c=0$ for all $k=0, \ldots, s-1$ iff $\langle\Lambda(e), \Lambda(c)\rangle=0$.

Proof: Since $\theta^{s}=1,\langle e, c\rangle=0$ is equivalent to

$$
\begin{aligned}
0 & =\sum_{j=0}^{l-1} e_{j}(x) \Omega\left(c_{j}(x)\right)=\sum_{j=0}^{l-1}\left(\sum_{i=0}^{s-1} e_{i j} x^{i}\right) \Omega\left(\sum_{k=0}^{s-1} c_{k j} x^{k}\right) \\
& =\sum_{j=0}^{l-1}\left(\sum_{i=0}^{s-1} e_{i j} x^{i}\right)\left(\sum_{k=0}^{s-1} \theta^{-1}\left(c_{k j}\right) x^{s-k}\right) \\
& =\sum_{j=0}^{l-1}\left(\sum_{j=0}^{l-1} \sum_{i=0}^{s-1} e_{i+h, j} \theta^{h}\left(c_{i j}\right)\right) x^{h}
\end{aligned}
$$

where the subscript $i+h$ is taken modulo $s$. Equating the coefficients of $x^{h}$ on both sides, we have $\sum_{j=0}^{l-1} \sum_{i=0}^{s-1} w_{i+h, j} \theta^{h}\left(c_{i j}\right)=0$, for all $0 \leq h \leq$ $s-1$. $\sum_{j=0}^{l-1} \sum_{i=0}^{s-1} e_{i+h, j} \theta^{h}\left(c_{i j}\right)=0$ is equivalent to $\theta^{h}\left(\nabla_{\theta, \lambda, l}^{s-h}(e) . c\right)=0$ which is further equivalent to $\nabla_{\theta, \lambda, l}^{s-h}(e, c)=0$, for all $0 \leq h \leq s-1$. Since $0 \leq h \leq$ $s-1$, condition is equivalent to $\left(\nabla_{\theta, \lambda, l}^{k}(e)\right) . c=0$ for all $k=0, \ldots, s-1$.

Corollary 67: Let $C$ be a skew quasi-constacyclic code of length $n=s l$ with index $l$ over $R$. Then $C^{\perp}=$ $\left\{a(x) \in M_{\theta, s}^{l}:\langle a(x), b(x)\rangle=0, \forall b(x) \in C\right\}$.

Corollary 68: Let $C$ be a skew quasi-constacyclic code of length $s l$ with index $l$ over $R$ and $\Lambda(C)$ be its image in $M_{\theta, s}^{l}$ under $\Lambda$. Then $\Lambda(C)^{\perp}=\Lambda\left(C^{\perp}\right)$, where the dual in $R^{s l}$ is taken with respect to the Euclidean inner product, while the dual in $M_{\theta, s}^{l}$ is taken with respect to the Hermitian inner product. The dual of a skew quasi-constacyclic code of length $s l$ with index $l$ over $R$ is a skew quasi-constacyclic code of length $s l$ with index $l$ over $R$.

Proposition 69: Let $\nabla_{\theta, \lambda, l}$ be skew quasi-constacyclic shift on $R^{n}$, let $\phi$ be the Gray map from $R^{n}$ to $Z_{3}^{3 n}$. Then $\phi \nabla_{\lambda, l}=\rho \phi \nabla_{\theta, \lambda, l}$, where $\rho(x, y, z)=(x, z, y)$ for every $x, y, z \in Z_{3}^{n}$. 50.

Proof: The proof is similar to the proof of Proposition

Theorem 70: The Gray image a skew quasi-constacyclic code over $R$ of length $n$ is permutation equivalent to the Gray image of a quasi-constacyclic code over $Z_{3}$ with length $3 n$.

Proof: The proof is similar to the proof of Theorem 51.

\section{1-Generator SKew QuASI-CONSTACYCLIC CODES OVER $R$}

A 1-generator skew quasi-constacyclic code over $R$ is a left $M_{\theta, s}$-submodule of $M_{\theta, s}^{l}$ generated by $\mathbf{f}(\mathbf{x})=$ $\left(f_{1}(x), f_{2}(x), \ldots, f_{l}(x)\right) \in M_{\theta, s}^{l}$ has the form $C=$ $\left\{g(x)\left(f_{1}(x), f_{2}(x), \ldots, f_{l}(x)\right): g(x) \in M_{\theta, s}\right\}$. Define the following map

$$
\Pi_{i}: M_{\theta, s}^{l} \longrightarrow M_{\theta, s}
$$

defined by $\left(e_{1}(x), e_{2}(x), \ldots, e_{l}(x)\right) \longmapsto e_{i}(x), 1 \leq i \leq l$. Let $\Pi_{i}(C)=C_{i}$. Since $C$ is a left $M_{\theta, s}$-submodule of $\bar{M}_{\theta, s}^{l}$, $C_{i}$ is a left $M_{\theta, s}$-submodule of $M_{\theta, s}$, that is a left ideal of $M_{\theta, s} . C_{i}$ is generated by $f_{i}(x)$. Hence $C_{i}$ is a principal skew constacyclic code of length $n$ over $R$. $f_{i}(x)$ is a monic right divisor of $x^{s}-\lambda$ that is $x^{s}-\lambda=h_{i}(x) f_{i}(x), 1 \leq i \leq l$.

A generator of $C$ has the form

$$
\mathbf{f}(\mathbf{x})=\left(g_{1}(x) f_{1}(x), g_{2}(x) f_{2}(x), \ldots, g_{l}(x) f_{l}(x)\right)
$$

where $g_{i}(x) \in R[x, \theta]$ such that $g_{i}(x)$ and $h_{i}(x)$ are right coprime for all $1 \leq i \leq l$.

Definition 71: Let $C=\left(g_{1}(x) f_{1}(x), g_{2}(x) f_{2}(x), \ldots, g_{l}(x)\right.$ $\left.f_{l}(x)\right)$ be a skew quasi-constacyclic code of length $n=s l$ with index $l$. Then unique monic polynomial

$f(x)=\operatorname{gcld}\left(\mathbf{f}(\mathbf{x}), x^{s}-\lambda\right)=\operatorname{gcld}\left(f_{1}(x), f_{2}(x), \ldots, f_{l}(x), x^{s}-\lambda\right)$

is called the generator polynomial of $C$.

Theorem 72: Let $C$ be a 1-generator skew quasiconstacyclic code of length $n=s 1$ with index $l$ over $R$ generated by $\mathbf{f}(\mathbf{x})=\left(f_{1}(x), f_{2}(x), \ldots, f_{l}(x)\right)$ where $f_{i}(x)$ is a monic divisor of $x^{s}-\lambda$. Then $C$ is a $R$-free code with rank $s-\operatorname{deg}(f(x))$ where $f(x)=\operatorname{gcld}\left(\mathbf{f}(\mathbf{x}), x^{s}-\lambda\right)$. Moreover, the set $\left\{\mathbf{f}(\mathbf{x}), x \mathbf{f}(\mathbf{x}), \ldots, x^{n-\operatorname{deg}(f(x))-1} \mathbf{f}(\mathbf{x})\right\}$ forms an $R$-basis of $C$.

Proof: Since $\operatorname{gcld}\left(f_{i}(x), x^{s}-\lambda\right)=m_{i}(x)$, it follows that $f(x)=\operatorname{gcld}\left(m_{1}(x), m_{2}(x), \ldots, m_{l}(x)\right)$ where $\Pi_{i}(C)=$ $\left(f_{i}(x)\right)=\left(m_{i}(x)\right)$ with $m_{i}(x) \mid\left(x^{s}-\lambda\right)$ for all $1 \leq i \leq l$. Let $c(x)=\sum_{i=0}^{n-k-1} c_{i} x^{i}$ and $c(x) \mathbf{f}(\mathbf{x})=0$. Then $\left(x^{s}-\right.$ $\lambda) \mid c(x) f_{i}(x)$ for all $1 \leq i \leq l$. Hence $\left(x^{s}-\lambda\right) \mid c(x) f_{i}(x) c_{i}(x)$ with $\operatorname{gcld}\left(c_{i}(x), \frac{x^{s}-\lambda}{f_{i}(x)}=1\right.$. That is $\frac{x^{s}-\lambda}{f_{i}(x)} \mid c(x)$ which implies that $\frac{x^{s}-\lambda}{f(x)} \mid c(x)$. Since $\operatorname{deg}\left(\frac{x^{s}-\lambda}{f(x)}\right)=s-k>$ $\operatorname{deg}(c(x))=n-k-1$, it is follows that $c(x)=0$. Thus, $\mathbf{f}(\mathbf{x}), x \mathbf{f}(\mathbf{x}), \ldots, x^{n-\operatorname{deg}(f(x))-1} \mathbf{f}(\mathbf{x})$ are $R$-linear independent. Further, $\mathbf{f}(\mathbf{x}), x \mathbf{f}(\mathbf{x}), \ldots, x^{n-\operatorname{deg}(f(x))-1} \mathbf{f}(\mathbf{x})$ generate $C$. So, $\left\{\mathbf{f}(\mathbf{x}), x \mathbf{f}(\mathbf{x}), \ldots, x^{n-\operatorname{deg}(f(x))-1} \mathbf{f}(\mathbf{x})\right\}$ forms an $R$-basis of $C$.

\section{CONCLUSION}

In this paper, we have introduced skew cyclic, skew quasicyclic, skew constacyclic and skew quasi-constacyclic codes over the finite ring $R$. By using the Gray map, we have studied the Gray images of cyclic, quasi-cyclic, constacyclic and their skew codes over $R$. We have obtained a representation of a linear code of length $n$ over $R$ using $C_{1}, C_{2}$ and $C_{3}$ which are linear codes of length $n$ over $Z_{3}$. We have obtained the parameters of quantum error-correcting codes from both cyclic and negacyclic codes over $R$. We have determined a sufficient condition for 1-generator skew quasi-constacyclic codes to be free. 


\section{ACKNOWLEDGMENT}

The authors would like to thank the anonymous referees for their valuable remarks and suggestions. We would like to thank S.T. Dougherty for helping to express a linear code $C$ of length $n$ over $R$ by means of three ternary codes and we would like to thank Sedat Akleylek for helping to find minimum distances by using computer program.

\section{REFERENCES}

[1] T. Abualrub, A. Ghrayeb, N. Aydın, I. Siap, On the construction of skew quasi-cyclic codes, IEEE Transsactions on Information Theory, 56 2081-2090, (2010).

[2] T. Abualrub, N. Aydın, P. Seneviratne, On $\theta$-cyclic codes over $F_{2}+v F_{2}$, Australasian Journal of Combinatorics, 54 115-126, (2012).

[3] M. Ashraf, G. Mohammad, Quantum codes from cyclic codes over $F_{3}+$ $v F_{3}$, International Journal of Quantum Information, 6 1450042, (2014).

[4] A. Bayram, I. Siap, Structure of codes over the ring $Z_{3}[v] /\left\langle v^{3}-v\right\rangle$, AAECC, DOI 10.1007/s00200-013-0208-x, (2013).

[5] M. Bhaintwal, Skew quasi-cyclic codes over Galois rings, Des. Codes Cryptogr., DOI 10.1007/s10623-011-9494-0.

[6] M. Bhaintwal, S. K. Wasan, On quasi-cyclic codes over $Z_{q}$ AAECC, 20 459-480, (2009).

[7] D. Boucher, W. Geiselmann, F. Ulmer, Skew cyclic codes, Appl. Algebra. Eng.Commun Comput., 18 379-389, (2007).

[8] D. Boucher, P. Sole, F. Ulmer, Skew constacyclic codes over Galois rings, Advance of Mathematics of Communications, 2 273-292, (2008).

[9] D. Boucher, F. Ulmer, Coding with skew polynomial rings, Journal of Symbolic Computation, 44 1644-1656, (2009).

[10] A. R. Calderbank, E.M.Rains, P.M.Shor, N.J.A.Sloane, Quantum error correction via codes over GF (4) ,IEEE Trans. Inf. Theory,44 13691387, (1998).

[11] Y. Cengellenmis, A. Dertli, S.T. Dougherty, Codes over an infinite family of rings with a Gray map, Designs, Codes and Cryptography, 72 559-580, (2014).

[12] A. Dertli, Y. Cengellenmis, S. Ere, On quantum codes obtained from cyclic codes over $A_{2}$, Int. J. Quantum Inform., 13 1550031, (2015).

[13] A. Dertli, Y. Cengellenmis, S. Eren, Quantum codes over the ring $F_{2}+$ $u F_{2}+u^{2} F_{2}+\ldots+u^{m} F_{2}$, nt. Journal of Alg., 3 115-121, (2015).

[14] J. Gao, Skew cyclic codes over $F_{p}+v F_{p}$, J. Appl. Math. \& Informatics, 31 337-342,(2013).

[15] J. Gao, L. Shen, F. W. Fu, Skew generalized quasi-cyclic codes over finite fields, arXiv: 1309.1621v1.

[16] M. Grassl, T. Beth, On optimal quantum codes, International Journal of Quantum Information, 2 55-64,(2004).

[17] A. R. Hammons, V. Kumar, A. R. Calderbank, N. J. A. Sloane, P. Sole, The $Z_{4}$-linearity of Kerdock, Preparata, Goethals and related codes, IEEE Trans. Inf. Theory, 40 301-319,(1994).

[18] S. Jitman, S. Ling, P. Udomkovanich, Skew constacyclic codes over finite chain rings, AIMS Journal.

[19] X.Kai, S.Zhu, Quaternary construction bof quantum codes from cyclic codes over $F_{4}+u F_{4}$, Int. J. Quantum Inform., 9 689-700, (2011).

[20] S. Ling, P. Sole, On the algebraic structures of quasi-cyclic codes I: finite fields, IEEE Trans. Inf. Theory, 47 2751-2760, (2001).

[21] S. Ling, P. Sole, On the algebraic structures of quasi-cyclic codes II: chain rings, Des.Codes Cryptogr., 30 113130, (2003).

[22] S. Ling, P. Sole, On the algebraic structures of quasi-cyclic codes III: generator theory, IEEE Trans. Inf. Theory, 51 2692-2000, (2005).

[23] Maheshanand, S. K. Wasan, On Quasi-cyclic Codes over Integer Residue Rings, AAECC, Lecture Notes in Computer Science, 4851 330336, (2007).

[24] J.Qian, Quantum codes from cyclic codes over $F_{2}+v F_{2}$, Journal of Inform.\& computational Science 6 1715-1722, (2013).

[25] J.Qian, W.Ma, W.Gou, Quantum codes from cyclic codes over finite ring, Int. J. Quantum Inform., 7 1277-1283, ( 2009).
[26] J. F. Qian, L. N. Zhang, S. X. Zhu, $(1+u)$-constacyclic and cyclic codes over $F_{2}+u F_{2}$, Applied Mathematics Letters, 19 820-823, (2006).

[27] I. Siap, T. Abualrub, N. Aydın, P. Seneviratne, Skew cyclic codes of arbitrary length, Int. Journal of Information and Coding Theory, (2010).

[28] P.W.Shor, Scheme for reducing decoherence in quantum memory, Phys. Rev. A., 52 2493-2496, (1995).

[29] A. M. Steane, Simple quantum error correcting codes, Phys. Rev. A., 54 4741-4751, (1996).

[30] M. Wu, Skew cyclic and quasi-cyclic codes of arbitrary length over Galois rings, International Journal of Algebra, 7 803-807,(2013).

[31] X.Yin, W.Ma, Gray Map And Quantum Codes Over The Ring $F_{2}+$ $u F_{2}+u^{2} F_{2}$, International Joint Conferences of IEEE TrustCom-11, (2011).

[32] S. Zhu, L. Wang, A class of constacyclic codes over $F_{p}+v F_{p}$ and their Gray images, Discrete Math. 311 2677-2682, (2011). 